Aus der Abteilung Neuroradiologie

(Prof. Dr. med. M. Knauth)

im Zentrum Radiologie

der Medizinischen Fakultät der Universität Göttingen

\title{
Die Rolle neuroprotektiver Faktoren beim akuten Schädelhirntrauma
}

\author{
INAUGURAL - DISSERTATION \\ zur Erlangung des Doktorgrades \\ der Medizinischen Fakultät \\ der Georg-August-Universität zu Göttingen
}

vorgelegt von

Nina Theysohn, geb. Koschnicke

aus

Düsseldorf

Göttingen 2012 
Dekan: Prof. Dr. med. M. P. Schön

I. Berichterstatter: Prof. Dr. med. M. Knauth

II. Berichterstatter: Prof. Dr. med. H. C. Ludwig

Tag der mündlichen Prüfung: 15.1.2013 


\section{Inhaltsverzeichnis}

Seite

1. Einleitung 5

1.1 Grundlagen des Schädelhirntraumas 5

1.1.1 Definition / Einteilung / Verletzungsarten 5

1.1.2 Epidemiologie 6

1.2 Diagnostik 7

1.3 Risikofaktoren für eine Nachblutung nach SHT 10

1.4 Neuroprotektive Faktoren 11

1.4.1 Protektiver Effekt weiblicher Geschlechtshormone 12

1.4.2 Erythropoietin und Neuroprotektion 13

1.4.3 Die Rolle der Gerinnungsmodulation bei der Neuroprotektion 14

1.5 Neuroprotektion beim ischämischen Hirninfarkt 15

1.5.1 Ischämische Präkonditionierung: TIA als neuroprotektiver Faktor 15

1.5.2 ASS als protektiver Faktor beim ischämischen Hirninfarkt 16

1.6 Anhalte für cerebrale Hypoxie nach SHT 17

1.7 Cerebrale Hypoxie als neuroprotektiver Faktor beim SHT im Tierversuch 19

1.8 Hypothese: Neuroprotektion beim ischämischen Infarkt ist übertragbar auf 19 das akute SHT

2. Beschreibung der Studie 21

2.1 Ziel 21

2.2 Charakterisierung der Beobachtungsgruppe 22

2.2.1 Ethikvotum 22

2.2.2 Einschlusskriterien 22

2.2.3 Ausschlusskriterien 22

2.3 Studiendesign 23

2.4 SHT-Fragebogen 23

3. Biometrie 24

3.1 Primärer Endpunkt 24

3.2 Sekundäre Endpunkte 24

3.3 Hypothesen 24

3.4 Statistische Auswertungsverfahren $\quad 25$

3.5 Biometrische Betreuung 25 
$\begin{array}{ll}3.6 \text { Dokumentation } & 26\end{array}$

4. Datenschutz 26

5. Ergebnisse $\quad 27$

5.1 Patientenerfassung, klinische und demographische Daten 27

5.2 Verteilung der initialen Blutungsraten und der Nachblutungen 30

5.3 Bedeutung des initialen GCS und des Patientenalters 30

5.4 Primäre und sekundäre Endpunkte 31

$\begin{array}{ll}\text { 6. Diskussion } & 33\end{array}$

7. Zusammenfassung 39

8. Literaturverzeichnis 41

9. Abkürzungen 53

10. Anhang: Fragebogen, GCS 54 


\section{Einleitung}

\subsection{Grundlagen des Schädelhirntraumas}

\subsubsection{Definition / Einteilung / Verletzungsarten}

Das akute Schädelhirntrauma (SHT) ist in den Industrieländern die häufigste Todesursache und der häufigste Grund für eine schwere Behinderung bei Patienten unter 45 Jahren (Rieger et al. 2002). In der klinischen Routine hat sich eine Einteilung des SHT in verschiedene Schweregrade anhand des Glasgow Coma Scale (GCS) durchgesetzt, da sie schnell und einfach zu erheben und gleichzeitig klinisch relevant ist (Anhang, Seite 57, Anlage 2). Mittels eines Punktesystems wird eine vereinheitlichte Einschätzung des Schweregrads eines neurologischen Defizits vorgenommen: Für 1. das Öffnen der Augen (Wachheit), 2. die motorischen Reaktionen und 3. die Bewusstseinslage wird jeweils ein definierter Punktwert vergeben - in der Summe zwischen 3 und 15 Punkten. An diesem Punktwert orientiert sich die Einteilung in ein leichtes (GCS 13-15), mittelschweres (GCS 9-12) und schweres (GCS 3 - 8) SHT. Eine weitere Einteilung erfolgt in SHT Grad 1-3. Hiernach bestehen bei Patienten bei einem SHT 1. Grades (Commotio cerebri) definitionsgemäß keine neurologischen Ausfälle, im Gegensatz zu den SHT 2. und 3. Grades (Contusio cerebri). Das SHT 2. Grades unterscheidet sich wiederum von einem SHT 3. Grades durch die Dauer einer bestehenden Vigilanzstörung: Verbessert sich die Bewusstseinslage des Patienten innerhalb der ersten 24 Stunden, spricht man definitionsgemäß von einem SHT 2. Grades; bei länger andauernden Vigilanzstörungen von einem SHT 3. Grades. Darüber hinaus unterscheidet man das geschlossene SHT, bei dem die harte Hirnhaut (Dura mater) intakt ist, von einem offenen SHT, bei dem eine Verletzung der Dura mater vorliegt. Eine Contusio cerebri kann verschiedene, sogenannte intra- oder extraaxiale Verletzungen verursachen. Extraaxial definiert eine außerhalb des Gehirngewebes lokalisierte Verletzung. Durch das Trauma können verschiedene Blutungen verursacht werden, welche auf Grund ihrer Lage zu den extraaxialen Verletzungen zählen: 
1. Epiduralhämatom (EDH): Hierbei handelt es sich um eine Einblutung zwischen der Dura mater und der Schädelkalotte, der typischerweise eine Verletzung der A. meningea media oder einer ihrer Äste zugrunde liegt.

2. Akutes Subduralhämatom (SDH): Dieses entsteht durch eine Verletzung der Brückenvenen und befindet sich zwischen Dura mater und sogenannter Spinnwebshaut (Arachnoidea).

3. Traumatische Subarachnoidalblutung (tSAB): Sie liegt zwischen Arachnoidea und Pia mater. Ihr liegt als Ursache eine Verletzung einer der Aa. cerebri oder einer anderen zum Circulus arteriosus Willisii gehörenden Arterien zu Grunde.

Als intraaxiale Verletzung wird eine Verletzung bezeichnet, bei der das Hirnparenchym selbst beschädigt wird, wobei sowohl die graue als auch die weiße Gehirnsubstanz betroffen sein können. Die Kontusionsblutung der grauen oder weißen Substanz beruht wahrscheinlich auf einer Paralyse und einer erhöhten Permeabilität eines traumatisierten Gefäßes. Hierbei können zusätzlich Nervenfasern entweder gedehnt oder auch abgerissen werden. Man spricht in diesem Fall von einer Scherverletzung (engl.: diffuse axonal injury, DAI) (Liu et al. 1999).

\subsubsection{Epidemiologie}

Nach den verfügbaren Angaben des Statistischen Bundesamtes wurden im Jahr 1996279.029 Patienten aufgrund eines SHT stationär behandelt. Bei einer Gesamtbevölkerung von 81.895.637 Einwohnern zu diesem Zeitpunkt ergibt sich eine Inzidenz von 340/100.000 Einwohner (Steudel et al. 2005). Insgesamt wird in der Literatur eine Inzidenz von 76 - 317/100.000 angegeben (Biberthaler et al. 2001; Wu et al. 2008). Die Mortalität des SHT betrug 1996 11,5/100.000 Einwohner, wobei die Altersgruppe $>75$ die höchste Mortalität aufwies, gefolgt von den 15- bis 25Jährigen. Der Anteil an SHT-bedingten Sterbefällen an allen verletzungsbedingten Sterbefällen lag bei $35,6 \%$ (Cortbus und Steudel 1999). Letale Folgen treten bei weniger als einem Prozent der leichten, bei $18 \%$ der mittelgradigen und bei nahezu $50 \%$ der schweren SHT auf (Rieger et al. 2002).

Bezüglich der Ursache des SHT gibt es keine bundesweite Datenerhebung. Nach einer Erhebung der Universitätsklinik Marburg wurde dort der Anteil der Verkehrsunfälle (Auto- und Fahrradunfälle) mit 39,9\% und der Stürze auf den Boden mit $41,8 \%$ an allen SHT errechnet (Koch 1996). 


\subsection{Diagnostik}

Da die Schnelligkeit der Einleitung einer adäquaten intensivmedizinischen bzw. neurochirurgischen Therapiemaßnahme in den ersten Stunden nach einem SHT richtungsweisend für die weitere Prognose des Patienten ist (Wiesmann und Bruckmann 1998), muss möglichst schnell eine präzise und schnelle Diagnostik erfolgen. Die Computertomographie des Neurokraniums (cCT) stellt hierbei das anerkannte und am weitesten verbreitete Standardverfahren dar. Hiermit werden die Verletzungen, die eine neurochirurgische Intervention notwendig machen, wie Impressionsfrakturen, epi- und subdurale Hämatome (Wiesmann und Bruckmann 1998; Zimmer und Reith 2008), mit ausreichender Sicherheit diagnostiziert.

Die Indikation für ein initiales cerebrales CT besteht bei einem GCS <15 und bei dem Vorliegen von Risikofaktoren (siehe 1.3, Seite 10) (Rieger et al. 2002; Saboori et al. 2007; Wiesmann und Bruckmann 1998). Die Vorteile der cCT gegenüber der sensitiveren Kernspintomographie bezüglich Blutungen und Scherverletzungen liegen bei der besseren Verfügbarkeit, der schnellen Diagnostik und der Möglichkeit für Intensivmediziner, die am Patienten installierten Überwachungs- und TherapieMaßnahmen beizubehalten. Zusätzlich kann eine schnelle und genaue Frakturdiagnostik erfolgen.

Die Limitationen der CCT zeigen sich zum Beispiel bei der Diagnostik von Scherverletzungen, da diese hier initial meist nur durch das gleichzeitige Auftreten von Einblutungen (Kontusionsblutungen) oder indirekten Zeichen wie Dichteminderungen mittels $\mathrm{CCT}$ diagnostiziert werden können. Fehlen diese Einblutungen oder sind diese so klein, dass sie der CT-Diagnostik entgehen, wird eine DAI auf Grund des Unfallhergangs (Akzelerations-/Dezelerationstrauma) oder auf Grund einer Diskrepanz zwischen Bildgebung und neurologischen Symptomen vermutet (Liu et al. 1999; Stiell et al. 2001; Wiesmann und Bruckmann 1998). Topal et al. fanden beispielsweise bei $12,5 \%$ der Patienten mit initial unauffälligem cCT nach mildem SHT DAI-suspekte Veränderungen in der Magnetresonananztomographie (MRT) (Topal et al. 2008).

Auch bestimmte Bereiche, wie z. B. der Hirnstamm oder Knochen-nah (Schädelbasis-nah) gelegene Anteile des Hirnparenchyms, können sich der sicheren Beurteilung entziehen, da an diesen Stellen Partialvolumeneffekte oder Aufhärtungsartefakte entstehen - diese Lokalisationen stellen allerdings 
Prädilektionsstellen für Kontusionen dar. Die MRT kann diese Nachteile der CT kompensieren und ist dieser hier überlegen, denn sowohl im Bereich des Hirnstamms als auch in Schädelbasis-nahen Regionen können Scherverletzungen und kleine Kontusionen dargestellt werden (Liu et al. 1999; Rieger et al. 2002). Dies gelingt unter anderem durch das Ausnutzen der paramagnetischen Eigenschaften der Blutabbauprodukte bei der sogenannten Suszeptibilitäts-gewichteten Darstellung im MRT (Susceptibility weighted imaging, SWI), oder etwas weniger sensitiv mittels T2*-gewichteten Sequenzen (Theysohn et al. 2011). Hier führen Mikroblutungen zu sogenannten Signalauslöschungen (Haacke et al. 2004; Haacke et al. 2009).

Die Darstellung der einzelnen Blutungstypen im initialen CT lässt sich wie folgt beschreiben: Das akute SDH liegt mehrheitlich supratentoriell, in ca. 15\% der Fälle bilateral. Auf Grund der anatomischen Gegebenheiten stellt es sich konkav und in der Akutphase hyperdens im Vergleich zum Hirnparenchym dar (Rieger et al. 2002).

Das EDH hingegen entsteht zu 90\% aufgrund von arteriellen Gefäßverletzungen im Rahmen einer Kalottenfraktur und befindet sich deshalb auch meistens auf der Seite der direkten Gewalteinwirkung, überwiegend unilateral und supratentoriell (95\%). Die Ausdehnung des EDH ist - im Gegensatz zum SDH - durch die Nahtstellen der einzelnen Schädelknochen, der sog. Suturen limitiert. Es resultiert eine bikonvexe Form.

Die traumatische SAB tritt meist in Kombination mit Hirnkontusionen und/oder Schertraumata auf und ist in der akuten Phase beispielsweise durch eine den Sulci folgende Hyperdensität gekennzeichnet (Mattioli et al. 2003; Wiesmann und Bruckmann 1998). Darüber hinaus kann sie sich in Umgebung der übrigen Liquorräume, typischerweise peri-mesenzephal, manifestieren.

Prädilektionsstellen für Hirnkontusionen, eventuell mit Einblutung, befinden sich fronto- oder temporo-polar. Die Ursache scheint in der Anatomie, der zerklüfteten Oberfläche der Schädelbasis und des Felsenbeins begründet. Kontusionen finden sich auf der Seite der Gewalteinwirkung (coup) oder auf der gegenüberliegenden Seite (contre coup). Computertomographisch zeigen sich sowohl hypodense Areale, die initial einem (Perifokal-)Ödem und im Verlauf nekrotischem Gewebe entsprechen, als auch hyperdense Anteile, denen Einblutungen zu Grunde liegen. Initial kann aber auch nur eine Hirnschwellung mit z.B. verstrichenen Hirnfurchen auftreten, oder es zeigen sich erste Anzeichen fokal verminderter Dichte (Wiesmann und Bruckmann 1998). 
Da eine Blutung im initialen CT, wie oben beschrieben, auch mit Latenz von Stunden bis Tagen auftreten kann (Lobato et al. 1991; McBride et al. 1993; Oertel et al. 2002), stellen sich die Fragen, ob, zu welchem Zeitpunkt und unter welchen Voraussetzungen das CT wiederholt werden sollte.

In einer Studie der UCLA (University of California, Los Angeles) wurden 142 Patienten mit moderatem oder schwerem SHT auf eine Vergrößerung ihrer im initialen CT festgestellten Hirnblutung untersucht. Dazu wurde innerhalb von 24 Stunden ein zweites CT durchgeführt. 42,3\% der beobachteten Gruppe zeigten eine Nachblutung, wobei $51 \%$ davon auf Kontusionsblutungen entfielen. Die Autoren sprachen sich deshalb für eine Notwendigkeit der frühen CT-Wiederholung aus (follow-up innerhalb der ersten 24 Stunden) (Oertel et al. 2002). Ähnliche Ergebnisse mit einer Nachblutungsrate von 23 - 47,5\% bei einem follow-up-CT innerhalb von 24 - 72 Stunden wurden auch in verschiedenen anderen Studien erzielt (McBride et al. 1993; Servadei et al. 2000; Stein SC et al. 1993). Brown et al. begründen zudem die Notwendigkeit von follow-up-cCTs in der Entscheidungshilfe zu einer sekundären Therapieänderung bei Veränderung des neurologischen Status. Hier wurde in $38 \%$ der Fälle auf Grund des cCTs eine medikamentöse oder chirurgische Intervention nötig (Brown et al. 2007).

Folgende Kriterien wurden für die Durchführung einer CT-Folgeuntersuchung aufgestellt:

1. Eine klinische Verschlechterung eines Patienten deutet auf eine progrediente intrakranielle Raumforderung hin und stellt somit eine Indikation dar.

2. Ein verzögertes Wachwerden oder eine verzögerte Aufwachreaktion nach Beendigung der Sedierung kann ein Hinweis für eine Nachblutung sein (Wiesmann und Bruckmann 1998).

Rieger et al. differenzieren etwas genauer, indem sie bei ihren Kriterien berücksichtigen, ob der Patient spontan atmet oder intubiert ist:

Beim nicht intubierten Patienten sollte generell, unabhängig vom initialen $\mathrm{CT}$, bei neurologischer Verschlechterung ein erneutes CT veranlasst werden. Ist der Patient jedoch neurologisch stabil, so ist das Kontroll-CT dennoch notwendig, falls ein EDH, SDH oder eine Kontusionsblutung im ersten CT zu sehen ist.

Bei intubierten Patienten wird ein Kontroll-CT im Fall eines initial unauffälligen Befundes bei polytraumatisierten Personen empfohlen. Fällt das initiale CT jedoch pathologisch aus, sollte nach einem stattgefundenen operativen Eingriff (cerebral 
oder anderweitig), bei auftretenden Hirndruckzeichen oder nach 24 Stunden eine Kontrolle erfolgen (Rieger et al. 2002).

Bezüglich der Indikationsstellung eines Kontroll-CTs bei Kindern wird in einer retrospektiven Studie von Schnellinger et al. zunächst eine sorgfältige, klinische Untersuchung gefordert, da eine sekundäre, chirurgische Intervention in nur einem Fall des Patientenkollektivs auf Grund des zweiten CTs nötig wurde (Schnellinger et al. 2010). Diese Vorgehensweise wird von weiteren Studien unterstützt, zumal die Nachblutungsrate bei Kindern in Abhängigkeit von der Blutungsart und des eingeschlossenen Kollektivs deutlich variiert (Hamilton et al. 2010; Schutzman et al. 1993).

\subsection{Risikofaktoren für eine Nachblutung nach SHT}

Da, wie oben dargestellt, ein initial unauffälliges CT beim akuten SHT eine sekundäre Parenchymblutung im weiteren Verlauf nicht ausschließt, sollte unter bestimmten Bedingungen ein erneutes Kontroll- CT erfolgen.

Bislang konnten verschiedene Faktoren identifiziert werden, welche den Therapieverlauf negativ beeinflussen und deren Vorhandensein für den Patienten ein erhöhtes Risiko für ein schlechtes Outcome darstellen.

Bekannte Risikofaktoren sind:

1. Alter:

Bei unter 50-Jährigen traten in $40 \%$ sekundäre Parenchymblutungen auf, während über 50-Jährige sogar zu $57 \%$ eine Nachblutung zeigten. Dies ist vermutlich auf degenerative Veränderungen wie erhöhte Fragilität und verminderte Dehnbarkeit der intrakraniellen Gefäße u.a. im Rahmen der Arteriosklerose z.B. infolge von Hypertonus und/oder Diabetes mellitus zurückzuführen (Oertel et al. 2002).

2. Geschlecht:

Oertel et al. stellten ebenfalls fest, dass, im Gegensatz zu $18 \%$ bei den Frauen, 48 $\%$ der Männer eine sekundäre Blutung entwickelten (Oertel et al. 2002). Dies lässt vermuten, dass das männliche Geschlecht einen weiteren Risikofaktor darstellt. Andere Interpretationsmöglichkeiten schließen protektive Effekte von weiblichen Geschlechtshormonen und eventuelle Unterschiede im Unfallhergang mit ein.

3. Antikoagulanzien: 
Ein anderer in der Literatur diskutierter Risikofaktor ist die Gerinnungshemmung mit verschiedenen Antikoagulanzien. Eine zum Unfallzeitpunkt bestehende Koagulopathie, zum Beispiel messbar durch eine verlängerte partielle Thromboplastinzeit (pTT), erhöht die Nachblutungsrate der Patienten nach einem SHT (Oertel et al. 2002; Stein SC et al. 1992). Eine erhöhte Mortalitätsrate von Patienten mit einer traumatischen intrakraniellen Verletzung nach regelmäßiger Marcumar-Einnahme wurde auch von Mina et al. festgestellt (Mina et al. 2002; Mina et al. 2003). Marcumar greift in die Gerinnung durch eine Antagonisierung des Vitamin $\mathrm{K}$ ein und hemmt dadurch die Produktion der Vitamin-K-abhängigen Gerinnungsfaktoren. Patienten mit einer International Normalized Ratio (INR) $\geq 2$, gleichbedeutend mit dem therapeutischen Bereich, scheinen jedoch das höchste Risiko für eine Nachblutung zu haben (Pieracci et al. 2007).

Es fielen allerdings auch Patienten mit einer Vormedikation mit Aspirin durch eine erhöhte Mortalitätsrate auf (Mina et al. 2002). Kennedy et al. und Wojcik kamen jedoch zu gegensätzlichen Ergebnissen, da sie bezüglich gerinnungshemmender Medikamente nach SHT keine Unterschiede zur Vergleichsgruppe feststellen konnten (Kennedy et al. 2000; Wojcik et al. 2001). Eine weitere Studie zeigte darüber hinaus keine statistisch signifikanten Unterschiede in der Art oder Frequenz der Hirnblutungen bei, mit Low-Dose-Aspirin therapierten SHT-Patienten gegenüber nicht vortherapierten (Spektor et al. 2003). In einer retrospektiven Studie von Baumgärtner et al. wurden die Krankenakten von 464 Patienten mit leichtem SHT ausgewertet und verschiedene Risikofaktoren für eine Blutung ermittelt. Dementsprechend wurden folgende Empfehlungen ausgesprochen: Bei einem Alter unter 15 und über 60 Jahren, einem Sturz als Unfallursache und einem Puls über 100/min sollte demnach eine 24-stündige Intensivüberwachung erfolgen, während bei Bewusstseinsstörungen, neurologischen Symptomen, auffälligem GCS, Koagulopathien und Antikoagulanzieneinnahme ein CT durchgeführt werden sollte (Baumgartner et al. 2002).

\subsection{Neuroprotektive Faktoren}

Vorgänge wie die Aktivierung der Entzündungskaskade, Apoptose, Exzitotoxizität oder oxidativer Stress nach akutem SHT weisen auf ein multifaktorielles Geschehen hin. Körpereigene, endogene Substanzen greifen in diese Vorgänge ein und können 
den klinischen Verlauf positiv beeinflussen. Diese neuroprotektiven Substanzen und Faktoren sowie deren Einfluss auf das Patientenoutcome sind vielversprechend (Schouten 2007). Hierunter werden verschiedene Faktoren diskutiert. Zum einen bestehen Hinweise auf den neuroprotektiven Effekt der weiblichen Geschlechtshormone (Berry et al. 2009; Cutler et al. 2007; Phelan et al. 2007) und die antioxidative Wirkung z. B. von Heat-Shock-Proteinen, welche eine Gewebsschädigung durch oxidativen Stress reduzieren sollen. Rekombinantes HSP 70 führte im Tierversuch nach fokaler, cerebraler Ischämie bereits zu einer Reduzierung des Infarktvolumens und einer verbesserten senso-motorischen Funktion (Zhan $X$ et al. 2010). Zudem existiert Evidenz bezüglich des neuroprotektiven Effekts des körpereigenen Hormons Erythropoietin (EPO). Zusätzlich soll im Weiteren auf die Rolle der Gerinnungsmodulation nach SHT eingegangen werden.

\subsubsection{Protektiver Effekt weiblicher Geschlechtshormone}

Nachdem bereits mehrere Studien „männliches Geschlecht“ als möglichen Risikofaktor identifiziert hatten (Oertel et al. 2002; Slewa-Younan et al. 2008), andere dies jedoch widerlegen (Mushkudiani et al. 2007), konnte für Progesteron ein positiver Effekt festgestellt werden (Cutler et al. 2007; Roof und Hall 2000; Stein DG und Hoffman 2003). Bei Ratten wurde die Ausbildung eines Perifokalödems um eine Kontusionsblutung vermindert (Pettus et al. 2005; Roof et al. 1996).

Das posttraumatische Hirnödem fiel dort in der mit Progesteron behandelten Gruppe bei beiden Geschlechtern kleiner aus als in der Vergleichsgruppe ohne ProgesteronSubstitution. Ein weiterer vermuteter Effekt ist die Reduktion der Entzündungskaskade und Apoptose (Pettus et al. 2005; Robertson et al. 2006).

Der neuroprotektive Effekt von Östrogenen auf ischämische Infarkte im Tiermodell konnte 1995 durch Hurn et al. nachgewiesen werden: Eine Vorbehandlung von Hasen mit 17ß-Estradiol verbesserte den intra-ischämischen cerebralen Blutfluss (Hurn et al. 1995). Aufgrund dieser Beobachtungen untersuchten Bramlett und Dietrich 2001 den Einfluss endogen zirkulierender weiblicher Geschlechtshormone auf ihren neuroprotektiven Effekt beim SHT. Es wurden weibliche Ratten mit ovariektomierten und männlichen Ratten verglichen; zusätzlich erzeugte man das SHT zu zwei unterschiedlichen Zykluszeitpunkten. Es zeigte sich, dass sowohl die 
histopathologische Kontusionsgröße als auch das Kontusionsvolumen in den einzelnen Gruppen unterschiedlich war. Die weiblichen Ratten wiesen im Vergleich zu den männlichen und den ovariektomierten weiblichen ein signifikant kleineres Kontusionsvolumen auf, die männlichen und ovariektomierten weiblichen untereinander unterschieden sich jedoch nicht. Der Zeitpunkt des induzierten SHT im Hinblick auf den weiblichen Zyklus spielte keine Rolle in Bezug auf die Kontusionsgröße (Bramlett und Dietrich 2001).

Weiterhin wurde in einer Studie von Wagner et al. untersucht, ob das Geschlecht und der weibliche Zyklus Einfluss auf die motorischen und kognitiven Fähigkeiten nach induziertem Schädelhirntauma haben. Analog zu der Studie von Hurn et al. zeigten auch hier die weiblichen Ratten posttraumatisch sowohl motorisch als auch in Kognitionstests bessere Ergebnisse. Der hormonelle Status zum Unfallzeitpunkt jedoch war von untergeordneter Rolle (Wagner et al. 2004).

\subsubsection{Erythropoietin und Neuroprotektion}

Eine andere langfristige Kompensation einer verminderten Sauerstoffversorgung stellt die Bildung des Hormons Erythropoietin dar, welches die Produktion von Erythrozyten anregt. Erythropoietin scheint zusätzlich selbst neuroprotektive Wirkungen zu besitzen. Im gesunden, adulten Gehirn kaum nachweisbar, wird das Erythropoietin-System im Rahmen einer cerebralen Hypoxie hochreguliert. Hier wirkt es unter anderem antiapoptotisch und antioxidativ. Vielversprechende Ergebnisse zeigte 2001 eine Göttinger Schlaganfall Studie, in der eine Erythropoietin-Therapie auf das Infarktvolumen und das neurologische Outcome nach A.-cerebri-mediaIschämie eine positive Wirkung hatte (Ehrenreich et al. 2002). In einer folgenden Multicenter Studie konnten diese Ergebnisse jedoch insbesondere in Zusammenhang mit einer rtPA (recombinant tissue Plasmonigen Activator) Therapie nicht reproduziert werden (Ehrenreich et al. 2009). Ähnliche Ergebnisse, jedoch angewandt auf SHT-Patienten, zeigte auch eine Pilotstudie, in der zunächst keine Anzeichen eines reduzierten Zelltods in der EPO-Gruppe festgestellt wurden (Nirula et al. 2010). Bereits in mehreren in-vivo-Studien konnten die beim ischämischen Infarkt beobachteten, protektiven Prozesse auf das akute SHT übertragen werden (Akdemir Ozisik et al. 2007; Lu et al. 2005). Diese Ergebnisse legen nahe, dass noch nicht alle Mechanismen der Erythropoietin-Wirkung und -Wechselwirkung bekannt sind. 


\subsubsection{Die Rolle der Gerinnungsmodulation bei der Neuroprotektion}

Da unmittelbar nach einem SHT, wenn nötig, die Gerinnungskaskade in Gang gesetzt wird und sich somit die Konzentrationen der einzelnen Gerinnungsfaktoren ändern, ist anzunehmen, dass die Beeinflussung der Gerinnung eine entscheidende Rolle bezüglich des klinischen Verlaufs, bedingt durch z.B. eine Nachblutung bei einem SHT-Patienten, einnimmt. Man vermutet bei einzelnen Gerinnungsfaktoren und in die Gerinnung eingreifenden Substanzen eine dosisabhängige Wirkung. Diese kann je nach Menge neuroprotektiv oder schädigend sein (Masada et al. 2000; Xi et al. 1999; Xi et al. 2000).

Einer dieser Gerinnungsfaktoren, dessen positiver oder negativer Einfluss noch diskutiert wird, ist Thrombin. Bei einem direkten Eintritt von Blut in das Hirnparenchym im Zuge eines SHT werden große Mengen von Prothrombin durch Faktor Xa in Thrombin umgewandelt (Xi et al. 2003). Aber auch ohne Blutung kommt es zur Erhöhung der Thrombinkonzentration nach einem SHT, da dort die Blut-HirnSchranke geschädigt wird und vermutlich Bestandteile der Gerinnungskaskade und somit auch Prothrombin eintreten können (Xi et al. 2002). Da jedoch die mRNA sowohl für Prothrombin (Dihanich et al. 1991) als auch für Faktor X (Shikamoto und Morita 1999) im Gehirn exprimiert und nach einem Trauma die Prothrombin-mRNA hochreguliert wird (Xi et al. 2002), ergibt sich die Vermutung, dass Thrombin auch ohne Schädigung der Blut-Hirn-Schranke gebildet werden kann.

Im in-vivo-Tierversuch bei Ratten verursacht eine hochdosierte Thrombininfusion ein Hirnödem in gleicher Weise wie eine intracerebrale Einbringung von $100 \mu \mathrm{L}$ Blut (Lee et al. 1996a; Lee et al. 1996b). Beim Menschen wurde eine negative Beeinflussung bei der Entstehung eines perihämorrhagischen Hirnödems durch Thrombin beschrieben (Hamada und Matsuoka 2000). Andererseits weist Thrombin auch eine antioxidative Wirkung und somit in geringer Konzentration auch eine neuroprotektive Komponente auf: in vitro konnte ein Schutz vor, den Zelltod induzierendem, oxidativem Stress nachgewiesen werden. Paradoxerweise vermindert eine in-vitroVorbehandlung von Nervenzellen mit niedrig dosiertem Thrombin die Zellschäden nach einer Hochdosisbehandlung mit Thrombin (Jiang et al. 2002). Diese Ergebnisse unterstützt eine in-vivo-Studie von $\mathrm{Xi}$ et al., bei der eine Thrombin-Vorbehandlung (thrombin preconditioning) den Hirnschaden nach z.B. einer Hirnblutung vermindert, 
indem die Hirnödembildung reduziert wird (Xi et al. 1999; Xi et al. 2000). Als Ursache für die Effekte der Thrombin-Präkonditionierung werden Aktivierung von ThrombinRezeptoren, Hochregulierung der Thrombin-Inhibitoren, wie z.B. PlasminogenAktivator-Inhibitor (PAl) oder Hitzeschockproteine (HSP) vermutet. Die unterschiedlichen Auswirkungen des Thrombins, ob neuroprotektiv oder hirnschädigend, lassen daher darauf schließen, dass mehrere Kaskaden beteiligt sind oder dosisabhängig die gleichen Signaltransduktionswege auf verschiedene Weise stimuliert werden.

\subsection{Neuroprotektion beim ischämischen Hirninfarkt}

Der ischämische Hirninfarkt führt oft zu einem ständigen Funktionsverlust des betroffenen Hirnparenchyms. Daher kommt der Behandlung eine zentrale Bedeutung zu: supportive Verfahren und Einflussfaktoren auf den postischämischen klinischen Verlauf sind von großem wissenschaftlichen Interesse, da sie zukünftig möglicherweise dazu beitragen können, Symptome nach einem Schlaganfall zu reduzieren.

\subsubsection{Ischämische Präkonditionierung: TIA als neuroprotektiver Faktor}

Die sogenannte ischämische Hypoxietoleranz beschreibt die natürliche Anpassung verschiedener Organgewebe an bestimmte Sauerstoffveränderungen. Im Fall einer sich langsam entwickelnden Hypoxie führt beispielsweise die Anregung der im Glomus caroticum befindlichen, sauerstoffempfindlichen Neurone zu einer Steigerung der Aktivität des kardio-pulmonalen Systems.

Bezüglich der sogenannten ischämischen Toleranz konnte schon in Studien am Herzen festgestellt werden, dass Angina pectoris nicht nur als Vorreiter eines Myokardinfarktes gilt, sondern dass diese vorübergehende Sauerstoffunterversorgung eine ischämische Präkonditionierung (IPC) des Herzmuskelgewebes darstellt. Bei Patienten mit Angina-pectoris (AP)-Anfällen nach einem Myokardinfarkt ist das nekrotische Areal kleiner als in der Vergleichsgruppe ohne AP in der Vorgeschichte (Andreotti et al. 1996; Evrengul et al. 2003; Murry et al. 1986; Ottani et al. 1995).

Kurzfristige Hypoxien eines Hirnareals, die einem bestimmten arteriellen Versorgungsgebiet zugeordnet werden können, aus denen kein bleibender 
neurologischer Schaden entsteht und deren Symptome sich definitionsgemäß innerhalb von 24 Stunden zurückbilden, werden transitorische ischämische Attacken (TIA) genannt. Diese können das erste Symptom einer kardiovaskulären Erkrankung sein und stellen ein Warnsignal eines eventuell bevorstehenden Infarkts dar: das Lebenszeitrisiko dieser Patienten, einen Schlaganfall zu erleiden, ist fünffach erhöht (Wilterdink et al. 1998). Eine klinische Studie erbrachte erste Hinweise, dass Schlaganfälle nach einer prodromalen, transitorisch ischämischen Attacke (TIA) mit geringeren neurologischen Defiziten überstanden werden als ohne (Weih et al. 1999). In der Rolle als möglicher neuroprotektiver Faktor in Bezug auf ischämische Hirninfarkte zeigte sich, dass in der Patientengruppe mit vorangegangenen TIAs das neurologische Defizit nach dem Hirninfarkt signifikant geringer war als in der Patientengruppe ohne TIA. Eine deutlich größere Studie konnte diese Ergebnisse reproduzieren (Moncayo et al. 2000): Eine TIA-induzierte, kurzzeitige Hypoxie kann als Stimulus für das Hirngewebe dienen, sich bei erneuter eventuell länger dauernder Hypoxie an diesen veränderten Zustand besser anzupassen. Welche Mechanismen genau zu einer Hypoxietoleranz führen, ist nicht im Einzelnen bekannt. Diese Ergebnisse legen nahe, dass eine IPC endogene protektive Vorgänge hervorrufen kann und somit das Gehirngewebe im Falle eines folgenden Insults bis zu einem gewissen Grad schützt.

Untermauert wurde dies 2002 in einem Tierversuch. Nach einer 15 minütigen Okklusion der A. cerebri media bei Ratten, gefolgt von einer dauerhaften Okklusion desselben Gefäßes drei Tage später, fiel die Hirnödem-Bildung signifikant geringer aus als in der Kontrollgruppe ohne vorherige entsprechende IPC (Masada et al. 2002). Darüber hinaus war dort sowohl die Schädigung der Blut-Hirn-Schranke als auch die Expression des HSP 70 vermindert. Letzteres deutet auf eine verminderte Stresssituation der Hirnzellen hin. Zuletzt wurden die zur Zeit diskutierten Mechanismen der ischämischen Präkonditionierung IPC in einem Review zusammengefasst (Durukan und Tatlisumak 2010).

\subsubsection{ASS als protektiver Faktor beim ischämischen Hirninfarkt}

Obwohl Acetylsalicylsäure (ASS) zur sekundären Prävention nach einem ischämischen Insult bereits lange etabliert ist, gelten die genauen Wirkungsmechanismen als noch nicht vollständig bekannt. 
Der bekannteste Effekt des ASS ist die Hemmung der Thrombozytenaggregation durch sein Eingreifen in die Bildung des Thromboxan A2 und damit die Hemmung der zellulären Gerinnung, wodurch scheinbar rheologische Eigenschaften verbessert und die Bildung von Emboli verringert werden. Dennoch wird vermutet, dass es darüber hinaus noch Thrombozyten-unabhängige Wirkungen des ASS bezüglich der Neuroprotektion gibt: In einer Studie von Grilli et al. konnte in vitro eine neuroprotektive Wirkung des ASS auch in Blutleere, das bedeutet auch ohne Anwesenheit von Thrombozyten, gezeigt werden. ASS wirkte sich protektiv auf die von Glutamat erzeugte Exzitotoxizität gegenüber Nervenzellen aus (Grilli et al. 1996). Diese unter Hypoxie entstandene, Gewebe schädigende Exzitotoxizität konnte auch durch Glutamatantagonisten aufgehoben werden (Meldrum 1990; Nellgard und Wieloch 1992; Simon et al. 1984). Die Neurotoxizität von extrazellulärem Glutamat oder seinen Agonisten wird durch eine Senkung des zelleigenen Energiemetabolismus und damit einen gestörten lonenaustausch bewirkt (Riepe et al. 1997a). Es konnte gezeigt werden, dass durch eine milde, selektive Enzymhemmung des Citratzyklus in den Mitochondrien der Energieverbrauch gesenkt wurde. Die Abnahme der Konzentration des Adenosintriphosphats (ATP) im Falle einer experimentell erzeugten schweren chemischen Hypoxie war daraufhin langsamer als ohne vorherige Hemmung und die freie Radikalbildung war geringer. Daraus folgte eine verbesserte postischämische neuronale Funktion (Riepe et al. 1996a); von ASS ist auch bekannt, dass es mit Mitochondrien interagiert. Riepe et al. zeigten, dass ASS den Verbrauch der intrazellulären Energieträger, wie ATP, nach induzierter Hypoxie verlangsamen kann. Auch die neuronale Funktion blieb erhalten. Sein Eingriff in die oxidative Phosphorylierung könnte also einen protektiven Effekt durch verbesserte endogene Hypoxietoleranz zur Folge haben (Riepe et al. 1997a). Dass IPC zu einer verbesserten mitochondrialen Integrität führt, ist bereits bekannt (Dave et al. 2001; Racay et al. 2007; Zhan RZ et al. 2002).

\subsection{Anhalte für cerebrale Hypoxie nach SHT}

In Umgebung traumatischer Gehirnschädigungen konnte inzwischen eine Minderdurchblutung nachgewiesen werden (Nordstrom et al. 2003). Hieraus resultiert die Hypothese der "traumatischen Penumbra“: Analog zum ischämischen Schlaganfall wird auch beim SHT von einer passageren Minderperfusion, 
insbesondere im Kontusionsareal ausgegangen, z.B. induziert durch Gefäßspasmen oder kompressionsbedingt auf Grund einer Hirnparenchymschwellung (Mayer et al. 1999). Möglicherweise ist diese Minderdurchblutung ein zusätzlicher Faktor, welcher den Verlauf des SHT beeinflusst.

Die Existenz perihämorrhagischer, ischämischer Läsionen, verursacht z.B. durch toxische Effekte, Apoptose oder kritische Reduktion des regionalen Blutflusses wird kontrovers diskutiert: Während Einige diese These stützen (Eriskat et al. 2000; Friedrich et al. 2000), hat eine andere Studie konträre Ergebnisse erbracht (Herweh et al. 2007). In diesem Punkt widersprechen sich auch klinische Studien (Schellinger et al. 2003; Zazulia et al. 2001). Ergebnisse einer PET-Studie von Zazulia et al. legen nahe, dass eine perihämorrhagische Oligämie sekundär durch einen reduzierten metabolischen Bedarf an Sauerstoff bei konstanter $\mathrm{O}_{2}$-Extraktionsfraktion bedingt ist und nicht eine direkt durch die intracerebrale Blutung (ICB) verursachte, pathologische Oligämie oder Ischämie darstellt (Zazulia et al. 2001). Eine Studie zeigte perihämorrhagische Perfusionsdefizite in perfusionsgewichteten Sequenzen (PWI) bei 9 akuten ICB-Patienten, die bei 3 der 9 Patienten eine beträchtliche Ausdehnung hatten, aber laut diffusionsgewichteten Sequenzen (DWI) nicht zu einer manifesten ischämischen Infarzierung führten (Schellinger et al. 2003). Diese Ergebnisse konnten im Tierversuch kürzlich bestätigt werden (Orakcioglu et al. 2008). Darüber hinaus konnten Furuya et al. eine lokale Minderperfusion von Hirngewebe nach SHT mithilfe der Xenon-CT nachweisen (Furuya et al. 2003). Weitere Hinweise auf die wichtige Rolle der Minderperfusion mit konsekutiver Hypoxie erbrachten Nordstrom et al. Sie konnten erhöhte Werte von Lactat in betroffenen Hirnarealen nachweisen (Nordstrom et al. 2003). In einer weiteren Studie wurden unmittelbar posttraumatisch noch normale Laktatwerte gemessen, welche nach 70-105 Minuten anstiegen (von 324+/48 auf 416+/-34 microM). Dieser Anstieg reicht jedoch alleine nicht aus, um als Gewebsazidose kausal die beobachtete sekundäre Zunahme des Defektes zu verursachen (Eriskat et al. 2000). Allgemein handelt es sich bei Laktat um ein Produkt der anaeroben Glykolyse und stellt somit einen Hinweis auf eine reduzierte Sauerstoffversorgung dar.

Aus den oben genannten Ergebnissen wird deutlich, dass die sogenannte peritraumatische Penumbra ein Gewebeareal repräsentiert, welches durch optimales Patientenmanagement positiv beeinflusst werden kann (Menon 2003). 


\subsection{Cerebrale Hypoxie als neuroprotektiver Faktor beim SHT im Tierversuch}

Die IPC gilt, wie zuvor beschrieben, als einer der stärksten neuroprotektiven Faktoren (Heusch und Schulz 1997; Heusch 2001; Yellon et al. 1998). Die Wirksamkeit konnte in vivo bereits in verschiedenen klinischen Studien im Zusammenhang mit Herz- und Hirninfarkten nachgewiesen werden (Moncayo et al. 2000; Ottani et al. 1995; Weih et al. 1999). Da eine lokale Minderperfusion beim SHT ebenfalls eine Rolle zu spielen scheint (Eriskat et al. 2000; Friedrich et al. 2000; Furuya et al. 2003; Nordstrom et al. 2003), wurde im Tierversuch untersucht, ob sich diese Erkenntnis analog auch auf das SHT übertragen lässt.

Dazu wurde in einer Studie von M.A. Perez-Pinzon 1999 bei Ratten mittels eines zwei-minütigen Verschlusses beider $A a$. carotides eine nicht letale, cerebrale Ischämie erzeugt. Nach 48 Stunden wurde sowohl bei der Versuchsgruppe mit IPC als auch bei einer Kontrollgruppe ein SHT induziert sowie anschließend eine histopathologische Untersuchung durchgeführt (Perez-Pinzon et al. 1999). Die dabei experimentell herbeigeführte, traumatische Läsion, in beiden Gruppen eine rechtshemisphärische Kontusion kranial der Capsula externa, wies in der IPCGruppe signifikant kleinere Volumina auf als in der Kontrollgruppe. Die Anzahl nekrotischer Neurone war in der IPC-Gruppe ebenfalls geringer. Obwohl dieser Unterschied bezüglich der Nervenzellschädigung nicht das Signifikanzniveau erreichte $(p<0,057)$, bestand ein klarer Trend (Perez-Pinzon et al. 1999).

Hieraus wurde gefolgert, dass ein subletaler ischämischer Hirninsult im Tierversuch die Toleranz gegenüber einem nachfolgenden moderaten SHT fördert.

\subsection{Hypothese: Neuroprotektion beim ischämischen Infarkt ist übertragbar auf das akute SHT}

Faktoren, für die eine neuroprotektive Wirkung beim akuten SHT diskutiert wird, sind unter anderem die antioxidative Wirkung von Hitzeschockproteinen, weibliche Geschlechtshormone und die Hormonwirkung des Erythropoietins (Bramlett und Dietrich 2001; Nirula et al. 2010; Pettus et al. 2005; Wagner et al. 2004; Zhan X et al. 2010).

Die positive Wirkung der IPC beim Hirninfarkt wurde sowohl im Tierversuch als auch in klinischen Studien mehrfach untermauert. Weiterhin existieren auch beim SHT post-kontusionelle und peri-hämorrhagische Oligämien oder Hypoxien (Furuya et al. 
2003; Nordstrom et al. 2003; Schellinger et al. 2003). Die Vermutung, dass analog zum ischämischen Infarkt eine peritraumatische, perihämorrhagische Penumbra existieren könnte, welche ebenfalls mittels IPC positiv beinflusst werden kann, konnte bereits im Tierversuch gezeigt werden (Perez-Pinzon et al. 1999). Anders als beim ischämischen Infarkt (Masada et al. 2002; Moncayo et al. 2000; Weih et al. 1999) existieren bisher jedoch keine systematischen, klinischen Untersuchungen dieses Phänomens beim akuten SHT.

Daher soll in dieser Arbeit die Hypothese überprüft werden, ob sich die experimentellen Daten zur Protektion analog zum ischämischen Schlaganfall auch auf das SHT übertragen lassen.

Weitere, in diesem Rahmen untersuchte, mögliche neuroprotektive Faktoren sind hier ASS, die Substitution weiblicher Geschlechtshormone und eine Vorbehandlung mit Erythropoietin. 


\section{Beschreibung der Studie}

\subsection{Ziel}

In dieser Studie sollen die o.g. Faktoren, welche sich bereits als potentiell neuroprotektiv herausgestellt haben, bezüglich ihres Einflusses auf das Outcome nach einem SHT untersucht werden. Diese werden für diese Studie in zwei Bereiche zusammengefasst:

1. IPC des Gehirns (mikroangiopathische Veränderungen des Hirnparenchyms in der Bildgebung / TIA in der Anamnese)

2. Neuroprotektive Substanzen (ASS, Östrogene / Progesteron)

In dieser retrospektiven Fallkontrollstudie wurden Daten zusammengetragen, die eine Aussage darüber machen sollten, ob eine IPC oder eine Prämedikation mit o.g. möglichen neuroprotektiven Substanzen beim SHT eine Rolle spielen.

Es wurden jeweils zwei Patientengruppen miteinander verglichen:

a) 1. Patienten mit SHT ohne cerebrale IPC.

2. Patienten mit SHT mit Zeichen einer cerebralen IPC.

b) 1. Patienten mit SHT ohne bestehende ASS-Medikation.

2. Patienten mit SHT und vorbestehender ASS-Medikation. 


\subsection{Charakterisierung der Beobachtungsgruppe}

\subsubsection{Ethikvotum}

Vor der Datenerhebung wurde ein positives Ethikvotum bezüglich dieser Studie eingeholt. Die Studie wurde gemäß der Deklaration von Helsinki durchgeführt.

\subsubsection{Einschlusskriterien}

Es wurden alle Patienten, die vom 1.1.1998 bis zum 31.12.2002 in der Notaufnahme (T/N, Schockraum) des Göttinger Universitätsklinikums aufgrund eines isolierten, geschlossenen SHT behandelt wurden, in die Studie eingeschlossen.

\subsubsection{Ausschlusskriterien}

Patienten mit einem im initialen CT bestehenden Hemisphäreninfarkt oder Einblutung in einen bereits vorbestehenden Infarkt-bedingten Parenchymdefekt mussten aus der Studie ausgeschlossen werden, da dort durch die schon bestehende Gewebeschädigung ein möglicher neuroprotektiver Effekt nicht beurteilt werden konnte. Dasselbe galt für Patienten, bei denen ein Zustand nach SHT mit vorbestehendem Parenchymdefekt bestand.

Ebenso nicht in die Studie eingeschlossen wurden Patienten, bei denen initial oder im Verlauf eine Sinus- oder Hirnvenenthrombose auftrat.

War die Ursache des SHT eine hypertone Massenblutung, so mussten diese Patienten ebenfalls ausgeschlossen werden.

Starben die Patienten innerhalb des ersten Tages, ohne dass ein follow-up-CT durchgeführt werden konnte, wurden ihre Daten nicht berücksichtigt.

Patienten, bei denen eine Anamneseerhebung, insbesondere bzgl. TIA oder ASS, nicht möglich war, z.B. auf Grund einer vorbestehenden Demenz, einer globalen Aphasie oder einer persistierenden Bewusstseinsstörung, wurden ausgeschlossen. Wurde fremdanamnestisch eine TIA gesichert oder zeigten sich im initialen CT mikroangiopathische Veränderungen des Hirnparenchyms, so wurden diese Patienten in die Gruppe der ischämisch Präkonditionierten aufgenommen. 
Patienten ohne Kontroll-Untersuchung (CT abgelehnt, nicht durchgeführt) bzw. ohne Verlaufsdaten (gegen ärztlichen Rat entlassen oder frühzeitig entlassen und Wiedervorstellungstermin nicht wahrgenommen) wurden ausgeschlossen.

\subsection{Studiendesign}

Die Studie wurde in Zusammenarbeit mit der Abteilung Medizinische Statistik der Medizinischen Fakultät der Universität Göttingen, namentlich Frau Dr. Kahler und Herrn Prof. Hilgers, geplant. In dieser Fallkontrollstudie wurden retrospektiv Krankenakten von Patienten ausgewertet, welche vor dem 31.12.2002 wegen eines Schädelhirntraumas in der Notaufnahme $(T / N)$ des Göttinger Universitätsklinikums behandelt wurden. Anhand eines vorher erstellten Fragebogens wurden zuerst das Notarztprotokoll und das Notaufnahmeformular ausgewertet und Name, Geburtsdatum, Traumahergang, Datum und Uhrzeit des Traumas sowie die Ankunft in der Notaufnahme, Befund des initialen CTs, ggf. stationäre Aufnahme (bzw. Notwendigkeit von intensivmedizinischer Überwachung), Blutdruck, ggf. Kreatininwert, vegetative Symptome und der initiale GCS dokumentiert. Aus der Krankenakte wurden Vorerkrankungen, Medikation und der Verlauf (follow-up-CT, Komplikationen, GCS) entnommen (Anhang, Seite 54, Anlage 1).

Darüber hinaus wurden die initialen CTs über die Leitstelle der Abteilung Neuroradiologie (Leitung: Herr Prof. Knauth) bestellt und an einem hierfür eingerichteten Arbeitsplatz gemeinsam von mir und einem erfahrenen Neuroradiologen (geblindet bzgl. Vorbefund) revidiert und im Falle einer Diskrepanz im Vergleich zum Vorbefund durch einen neuroradiologischen Facharzt zusätzlich evaluiert („Consensus-Rating“).

Nach Eingabe in eine Datenbankanwendung (MS Excel) erfolgte die statistische Auswertung.

\subsection{SHT-Fragebogen}

Siehe Anhang, Seite 54, Anlage 1. 


\section{Biometrie}

Die Daten wurden in eine Datenbankanwendung (MS Excel) eingegeben und pseudonymisiert. Anschließend wurden die Daten anhand der Parameter: IPC JA oder NEIN und ASS Einnahme vor dem Unfall JA oder NEIN eingeteilt - aufgrund der zu geringen Fallzahl konnten bzgl. der Hormoneinnahme und der Erythropoietinbehandlung keine auswertbaren Gruppen gebildet werden.

\subsection{Primärer Endpunkt}

Als primärer Endpunkt wurde der Nachweis einer akuten Kontusionsblutung in der Nachfolge-CT (Kontroll- CT) gewählt.

\subsection{Sekundäre Endpunkte}

Als sekundäre Endpunkte wurden gewählt:

1. Der GCS bei Entlassung bzw. nach 3 Tagen zur Beurteilung des klinisch neurologischen Verlaufs der Patienten. Der Wert wurde jeweils aus den intensivmedizinischen bzw. stationären Verlaufsdokumentationsbögen entnommen.

2. Komplikationen während des stationären Aufenthalts

3. Tod des Patienten

\subsection{Hypothesen}

Aus einer tierexperimentellen Arbeit ist bereits hervorgegangen, dass durch eine künstlich erzeugte, nicht tödliche, kurze Ischämie des Gehirns 48 Stunden vor einem folgenden SHT die Kontusionsgröße verringert wurde (Perez-Pinzon et al. 1999). Die Anzahl der nekrotischen Zellen in dieser Gruppe war ebenfalls um $63 \%$ geringer.

In verschiedenen klinischen Studien konnte gezeigt werden, dass eine lokale Minderperfusion beim SHT eine Rolle zu spielen scheint (Eriskat et al. 2000; Friedrich et al. 2000; Furuya et al. 2003; Nordstrom et al. 2003). Bezüglich ASS ist inzwischen bekannt, dass die Einnahme bei Nervenzellen eine verbesserte endogene Hypoxietoleranz zur Folge hat (Riepe et al. 1997a).

Die primären Hypothesen lauten: 
1. Patienten mit einer vorbestehenden IPC im Vergleich zu Patienten ohne eine solche weisen bei einem folgenden SHT eine geringere Inzidenz an sekundären „aufblühenden“ Kontusionsblutungen auf.

2. Patienten mit ASS-Medikation vor einem SHT im Vergleich zu Patienten ohne vorherige ASS-Einnahme weisen ebenfalls eine geringere Nachblutungsrate auf (primärer Endpunkt).

3. Das Vorkommen einer Nachblutung sollte bei Östrogen/Progesteron-Einnahme ebenfalls vermindert sein.

Aufgrund des geringeren Ausmaßes der Kontusionsblutungen sollte es auch zu einem besseren GCS im Verlauf, zu einem komplikationsloseren stationären Aufenthalt und zu weniger Todesfällen kommen (sekundäre Endpunkte).

\subsection{Statistische Auswertungsverfahren}

Für alle Zielgrößen wurden die Befunde zunächst exploratorisch untersucht und deskriptiv ausgewertet. Dokumente und personenbezogene Daten wurden streng vertraulich behandelt. Hierzu wurden die üblichen Verfahren wie SPSS und/oder Statistica genutzt.

Als parametrisches Testverfahren kam in erster Linie bei metrisch skalierten Daten der (Student) t-Test zur Anwendung. Es wurde eine Varianzanalyse (ANOVA) einschließlich Bonferroni-Korrektur für multiple Vergleiche durchgeführt.

Zur Feststellung möglicher, dem beobachteten Effekt zugrundeliegender Effekte wurde eine multivariate Analyse eingesetzt. Kamen Prüfungen von Raten (Nachblutung) in Frage, so ist dafür der Chi-Quadrat-Test (qualitative Parameter) herangezogen worden.

Für die numerischen Variablen (GCS) erfolgte der Vergleich der Medianwerte.

\subsection{Biometrische Betreuung}

Freundlicherweise übernahmen Frau Dr. Kahler und Herr Prof. Hilgers - Abteilung Medizinische Statistik, Medizinische Fakultät der Georg-August-Universität Göttingen - die Biometrische Betreuung und die Planung des Stichprobenumfangs. 


\subsection{Dokumentation}

Die Dokumentation erfolgte auf standardisierten Formularen vor Eingabe in die Datenbank.

\section{Datenschutz}

Die Daten sind nach der Erhebung der Score-Werte pseudonymisiert worden und werden ausschließlich anonym veröffentlicht. 


\section{Ergebnisse}

\subsection{Patientenerfassung, klinische und demographische Daten}

Es wurden die Daten von insgesamt 260 Patienten ausgewertet, von denen 120 aus den folgenden Gründen ausgeschlossen wurden:

- bei 34 Patienten fehlte das follow-up-CT;

- 9 Patienten starben am ersten Tag ohne durchgeführtes follow-up-CT;

- bei 42 Patienten konnte keine Anamnese erhoben werden;

- 7 Patienten zeigten eine Einblutung in einen vorbestehenden, Infarkt-bedingten Parenchymdefekt;

- 5 Patienten hatten einen vorbestehenden Parenchymdefekt durch Z. n. SHT;

- bei 6 Patienten lag eine Sinus- oder Hirnvenenthrombose vor;

- 11 Patienten zeigten einen territorialen Infarkt im initialen CT;

- 6 Patienten erlitten das SHT in Folge einer hypertonen Massenblutung.

Die Analyse umfasste schließlich 140 SHT-Patienten (im Alter von 6 bis 91 Jahren, das Durchschnittsalter betrug 54,6 Jahre, 92 Männer und 48 Frauen).

Die für das SHT ursächlichen Unfallmechanismen waren vornehmlich Stürze unterschiedlicher Genese $(n=94)$, Stürze aus der Höhe $(n=8)$, PKW- $(n=22)$, Motorrad- $(n=4)$ und Fahrradunfälle $(n=12)$. Unter Alkoholeinfluss standen $25,7 \%$ der Patienten ( $n=36$ ). Ca. $21 \%$ der Patienten litten unter Übelkeit und Erbrechen zum Zeitpunkt der ärztlichen Untersuchung. Neben den unten näher erläuterten Nachblutungsraten sowie unterschiedlichsten neurologischen Symptomatiken traten im Verlauf intrakranielle Komplikationen wie Meningitiden / Ventrikulitiden ( $n=3)$, maligne Hirnödeme $(n=2)$, Krampfanfälle $(n=5)$, Hydrocephalus internus $(n=2)$ oder Hirnorganische Psychosyndrome $(n=3)$ auf. Systemische Komplikationen waren vornehmlich Pneumonien $(n=23)$, bei jeweils einem Patienten wurde eine Lungenembolie und ein Lungenödem diagnostiziert. 11 Patienten verstarben im Verlauf des klinischen Aufenthaltes (Tabelle 1). Bezüglich des Durchschnittsalters der Patienten gruppiert nach Unfallhergang ist hervorzuheben, dass dieses bei PKWUnfällen $(28,5$ Jahre, SD 12,8) deutlich niedriger lag als bei Stürzen aus dem Stand (60,1 Jahre, SD 18,2), Treppenstürzen (65,1 Jahre, SD 16,2) oder Synkopen (68,8 Jahre, SD 14,6) (Grafik 1). 
Tabelle 1: klinische und demographische Daten

Patientenzahl Anteil (\%)

\begin{tabular}{|c|c|c|}
\hline & 140 & 100 \\
\hline \multicolumn{3}{|l|}{ Alter (Jahre) } \\
\hline $0-15$ & 3 & 2,1 \\
\hline $16-30$ & 20 & 14,3 \\
\hline $31-45$ & 24 & 17,1 \\
\hline $46-60$ & 32 & 22,9 \\
\hline $61-75$ & 33 & 23,6 \\
\hline$>76$ & 28 & 20 \\
\hline \multicolumn{3}{|l|}{ Geschlecht } \\
\hline Männlich & 92 & 65,7 \\
\hline Weiblich & 48 & 34,3 \\
\hline \multicolumn{3}{|l|}{ Unfallmechanismus } \\
\hline Sturz aus Höhe & 8 & 5,7 \\
\hline Sturz aus dem Stand & 66 & 47,1 \\
\hline Synkope & 12 & 8,6 \\
\hline Treppensturz & 16 & 11,4 \\
\hline Fahrradunfall & 12 & 8,6 \\
\hline Motorradunfall & 4 & 2,9 \\
\hline PKW-Unfall & 22 & 15,7 \\
\hline Alkoholisiert & 36 & 25,7 \\
\hline Übelkeit/Erbrechen bei Aufnahme & 29 & 20,7 \\
\hline \multicolumn{3}{|l|}{ Komplikationen intrakraniell } \\
\hline Nachblutung & 50 & 35,7 \\
\hline Meningitis/Ventrikulitis & 3 & 2,1 \\
\hline Malignes Hirnödem & 2 & 1,4 \\
\hline Hydrocephalus internus & 2 & 1,4 \\
\hline Krampfanfälle & 5 & 3,6 \\
\hline HOPS & 3 & 2,1 \\
\hline \multicolumn{3}{|l|}{ Komplikation systemisch } \\
\hline Lungenembolie & 1 & 1,4 \\
\hline Lungenödem & 1 & 1,4 \\
\hline Pneumonie & 23 & 16,4 \\
\hline Tod & 12 & 8,6 \\
\hline
\end{tabular}


Grafik 1: Mittelwert und Standardabweichung des durchschnittlichen Patientenalters bezogen auf die verschiedenen Unfallmechanismen (y-Achse: Patientenalter in Jahren, x-Achse: Unfallmechanismus).

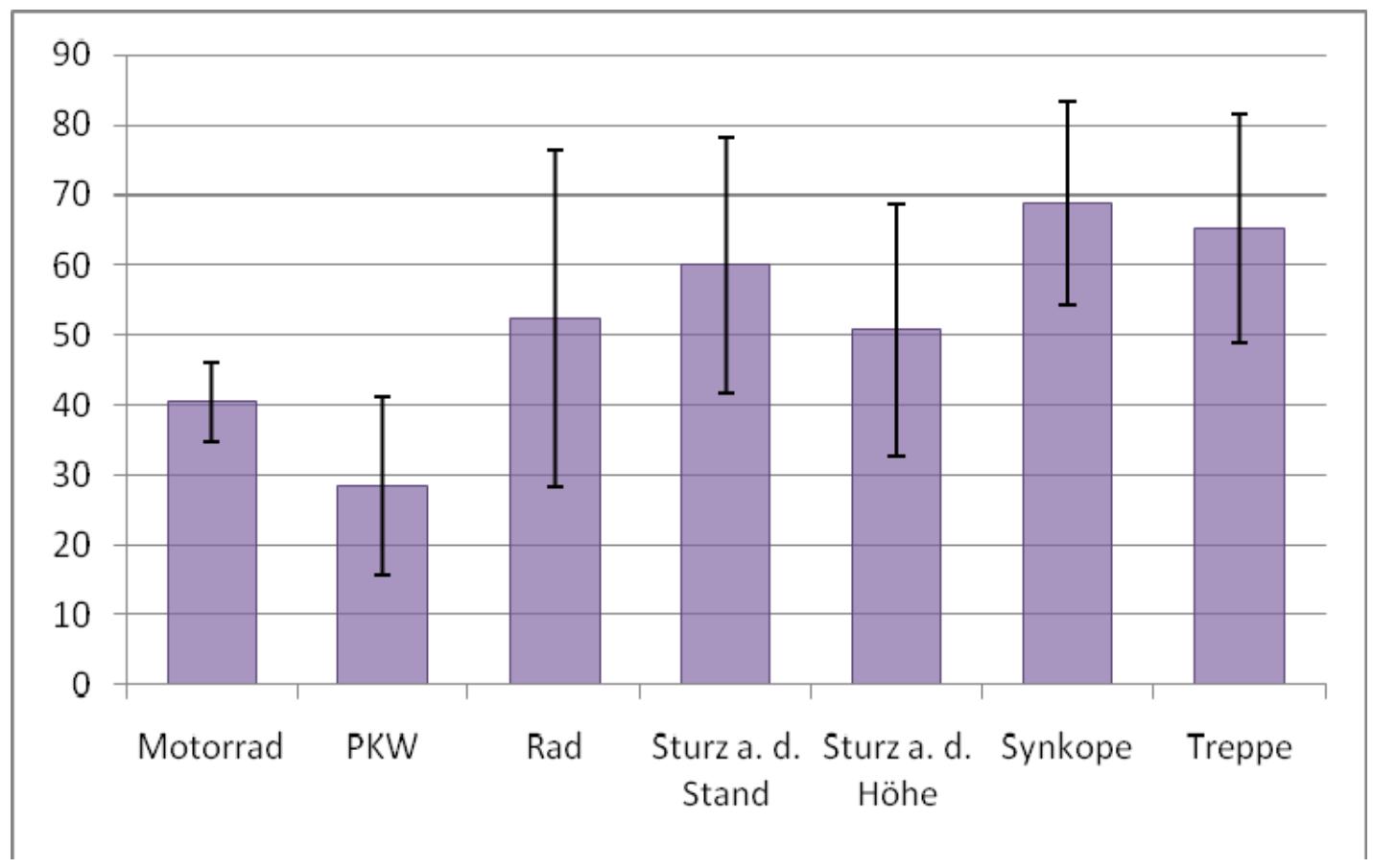




\subsection{Verteilung der initialen Blutungsraten und der Nachblutungen}

Im initialen CCT unmittelbar nach der Aufnahme wiesen 99 Patienten (71\%) bereits eine oder mehrere Kontusionsblutungen auf, während bei 41 Patienten (29\%) das initiale CCT diesbezüglich keinen pathologischen Befund aufwies (Tabelle 2). Im weiteren Verlauf konnten in den CCT-Kontrollen der bereits Kontusionsblutungen aufweisenden 28 Patienten (28\%), in der anfänglich unauffälligen cCT-Gruppe 22 aufblühende Kontusionen bzw. Nachblutungen (54\%) nachgewiesen werden (Tabelle 2). Es wurden bei 33\% (16/48) der weiblichen und bei 37\% (34/92) der männlichen Patienten Nachblutungen festgestellt.

Tabelle 2: Nachblutungsraten

\begin{tabular}{lll}
\hline & ja (\%) & nein (\%) \\
\hline Kontusionen im initialen CT & $99(71 \%)$ & $41(29 \%)$ \\
Nachblutung / Aufblühen einer Kontusion & $28(28 \%)$ & $22(54 \%)$ \\
\hline
\end{tabular}

\subsection{Bedeutung des initialen GCS und des Patientenalters}

Bei Patienten mit initial niedrigem GCS traten im Verlauf in mehr als der Hälfte (55\%) der Fälle klinische Verschlechterungen auf. Bei Patienten mit initial unauffälligem CT und GCS traten noch bei 16\% $(n=8)$ Befundverschlechterungen und 14\% $(n=7)$ Kontusionsblutungen auf. Der Vergleich von Patienten unter und über 60 Jahren zeigte sowohl eine häufigere klinische Verschlechterung als auch eine höhere Nachblutungsrate bei Patienten unter 60 Jahren (Tabelle 3).

Tabelle 3: klinische Verschlechterungen und Nachblutungsraten in Abhängigkeit von Alter und initialem GCS-Score

\begin{tabular}{lcc}
\hline & klinische Verschlechterung & Nachblutungen \\
\hline GCS 3-5* & $55 \%$ & $20 \%$ \\
GCS 6-10 & $50 \%$ & $19 \%$ \\
GCS $11-14$ & $16 \%$ & $14 \%$ \\
& & \\
Alter $<60(n=79)$ & $14 \%(n=11)$ & $41 \%(n=32)$ \\
Alter $>60(n=61)$ & $20 \%(n=12)$ & $30 \%(n=18)$ \\
\hline
\end{tabular}

*Tod wurde auch bei initialem GCS von 3 als klinische Verschlechterung gewertet. 


\subsection{Primäre und sekundäre Endpunkte}

Insgesamt kam es bei 35,7\% zum „Aufblühen“ von Kontusionen. Während bei Patienten mit cerebraler Ischämie in der Anamnese jeder Zehnte (10,4\%) eine Nachblutung aufwies, zeigte sich in der Kontrollgruppe ohne Vorschädigung bei fast jedem Zweiten (48,9\%) eine Nachblutung (Tabelle 4, Grafik 2).

Bei der ASS einnehmenden Gruppe traten in 19,5\% Nachblutungen auf, während die Kontrollgruppe zu 42,4\% solche aufwies. In der multivariaten Analyse konnte für die ASS Gruppe bezüglich der Nachblutungsrate jedoch kein signifikantes Ergebnis beobachtet werden ( $p=0,378$ multivariat) (Tabelle 5).

Die zusätzliche Berücksichtigung des GCS-Verlaufs erbrachte keine statistisch signifikanten Ergebnisse. Der GCS-Mittelwert sowohl in der ASS- $(12,4)$ als auch in der IPC-Gruppe $(12,9)$ unterschied sich nicht signifikant von den Kontrollgruppen (12,9 und 12,7). Auch der Anteil der Todesfälle war in beiden Gruppen etwa gleich.

Bei 7 Patienten wurde die Einnahme von Östrogenen / Progesteron dokumentiert oder konnte im Nachhinein anamnestisch erhoben werden. Auf Grund der niedrigen Fallzahl konnte jedoch eine statistische Auswertung nicht erfolgen. Gleiches gilt für die Einnahme von Erythropoietin, welches nur bei einem Patienten dokumentiert werden konnte.

Tabelle 4: Primäre und sekundäre Endpunkte in Bezug auf eine cerebrale ischämische Präkonditionierung (uni- und multivariat).

\begin{tabular}{|c|c|c|c|}
\hline & \multicolumn{2}{|c|}{ ischäm. Präkonditionierung } & \multirow[b]{2}{*}{ p-Wert } \\
\hline & $(+)$ & $(-)$ & \\
\hline \multirow{2}{*}{ Nachblutungsrate $(n=50)$} & $5 / 48(10,4 \%)$ & $45 / 92(48,9 \%)$ & $<0,001$ (univariat) \\
\hline & & & $<0,001$ (multivariat) \\
\hline GCS-Mittelwert (Aufnahme) & 12,02 & 11,01 & n. signifikant \\
\hline GCS-Mittelwert (Tag 3) & 12,13 & 11,47 & n. signifikant \\
\hline GCS-Mittelwert (Entlassung) & 12,9 & 12,7 & n. signifikant \\
\hline Anzahl der Todesfälle $(n=12)$ & $4 / 48(8,3 \%)$ & $8 / 92(8,7 \%)$ & n. signifikant \\
\hline
\end{tabular}


Grafik 2: Vergleich der Nachblutungen bei vorhandener und fehlender IPC. Die Gruppe der nicht präkonditionierten ist mit $n=92$ Patienten etwa doppelt so groß wie die Gruppe der ischämisch präkonditionierten Patienten $(n=48)$. Eine sekundäre Blutung erlitten 5/48 (10 \% der IPC-Gruppe) und 45/92 (49\% der Nicht-IPC-Gruppe).

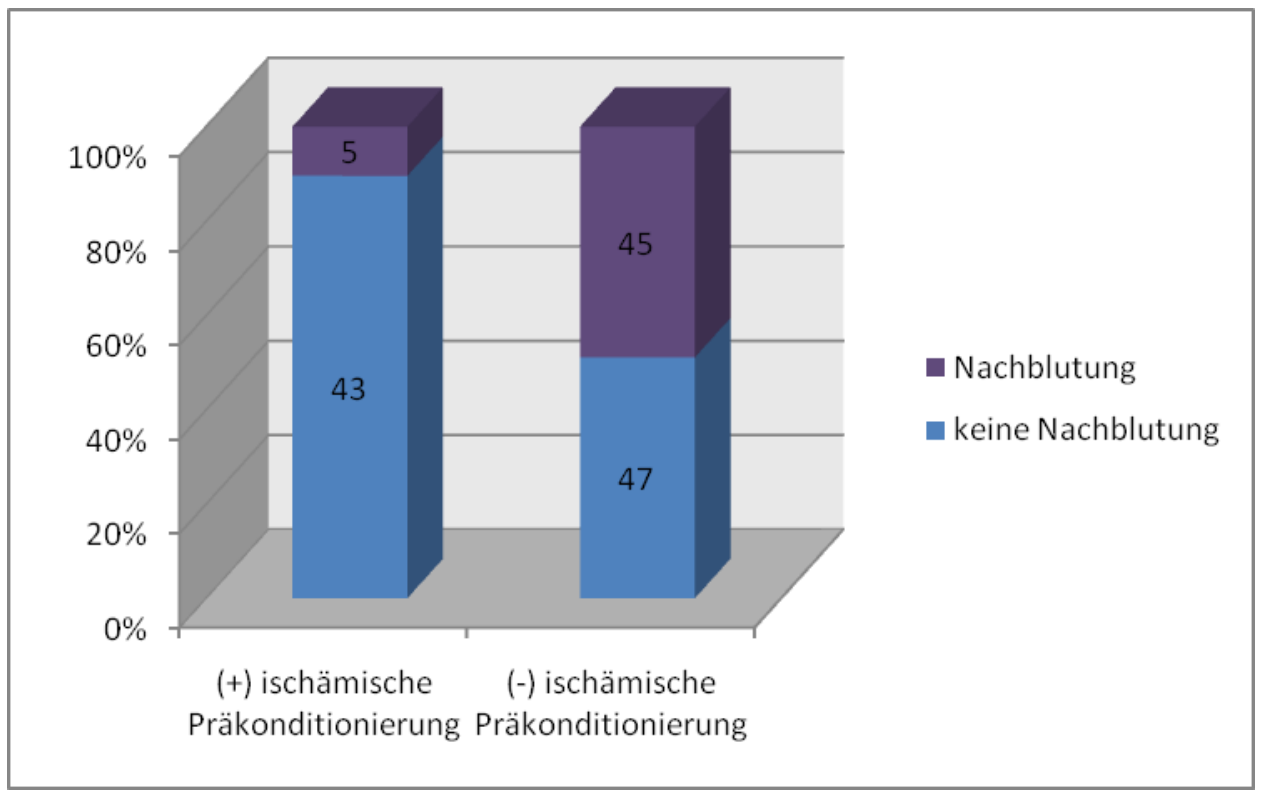

Tabelle 5: Primäre und sekundäre Endpunkte in Bezug auf die ASS-Einnahme (uni- und multivariat).

ASS-Medikation

\begin{tabular}{lccc} 
& ASS-Medikation & \\
& $(+)$ & $(-)$ & p-Wert \\
\hline Nachblutungsrate ( $\mathrm{n}=50)$ & $8 / 41(19,5 \%)$ & $42 / 99(42,4 \%)$ & 0,0206 (univariat) \\
& & & 0,378 (multivariat) \\
GCS-Mittelwert (Aufnahme) & 11,9 & 11,2 & n. signifikant \\
GCS-Mittelwert (Tag 3) & 11,63 & 11,71 & n. signifikant \\
GCS-Mittelwert (Entlassung) & 12,4 & 12,9 & n. signifikant \\
Anzahl der Todesfälle ( $\mathrm{n=12)}$ & $4 / 41(9,8 \%)$ & $8 / 99(8,1 \%)$ & n. signifikant \\
\hline
\end{tabular}




\section{Diskussion}

In Analogie zum ischämischen Hirninfarkt und seiner ischämischen Penumbra gibt es Hinweise auf eine sogenannte "traumatische Penumbra“ im Kontusionsareal nach SHT (Nordstrom et al. 2003; Orakcioglu et al. 2008; Schellinger et al. 2003). Hier wird die Existenz perihämorrhagischer, ischämischer Läsionen diskutiert (Furuya et al. 2003; Nordstrom et al. 2003), deren Ausmaß und Therapie den klinischen Verlauf des Patienten nach SHT beeinflussen könnten (Menon 2003).

Eine kurze ischämische Episode, die nicht zum Zelluntergang führt, schützt das Gewebe vor einer länger andauernden, ansonsten zum Gewebeuntergang führenden Ischämie. Dieses als ischemic tolerance bezeichnete Phänomen wurde sowohl am Myokard durch Angina-pectoris-Episoden (Murry et al. 1986) als auch am menschlichen Gehirn durch eine TIA (Weih et al. 1999) beschrieben und stellt einen wesentlichen endogenen adaptiven Mechanismus gegen eine Hypoxie dar. Ausgehend von der Hypothese einer "traumatischen Penumbra“ wurde hier untersucht, ob sich eine IPC beim Menschen protektiv auf ein folgendes SHT auswirken kann. Diese Hypothese konnte bereits in einer tierexperimentellen Studie (Perez-Pinzon et al. 1999) bekräftigt werden.

In der vorliegenden Studie sollte untersucht werden, ob Patienten, welche eine TIA in der Anamnese erlitten oder bildmorphologisch relevante mikroangiopathische Veränderungen im CT aufwiesen, zusammengefasst als potentielle IPC des Gehirns, eine geringere Nachblutungsrate nach einem SHT aufwiesen als die Kontrollgruppe. Als zweiter potentiell neuroprotektiver Faktor sollte die Auswirkung einer dem SHT vorhergehenden ASS-Einnahme auf die Nachblutungsrate geprüft werden. ASS könnte, wie oben beschrieben, die endogene Hypoxietoleranz des Hirngewebes verbessern (Riepe et al. 1997a). Weitere in der Literatur diskutierte neuroprotektive Faktoren, wie weibliche Geschlechtshormone (Bramlett und Dietrich 2001; Pettus et al. 2005; Robertson et al. 2006; Roof und Hall 2000) und das Hormon Erythropoietin (Akdemir Ozisik et al. 2007; Lu et al. 2005) konnten auf Grund geringer Fallzahlen nicht weiter verfolgt werden.

Als primärer Endpunkt wurde deshalb das Auftreten von Nachblutungen (Kontusionsblutungen) bzw. das „Aufblühen“ schon bestehender Kontusionsblutungen gewählt, welche anhand des CTs bei Aufnahme und in nachfolgenden Kontroll-CTs diagnostiziert wurden. Die initialen CTs wurden an 
einem hierfür eingerichteten Arbeitsplatz gemeinsam von mir und einem erfahrenen Neuroradiologen (geblindet bzgl. Vorbefund) beurteilt und im Falle einer Diskrepanz im Vergleich zum Vorbefund durch einen neuroradiologischen Facharzt zusätzlich evaluiert und im Konsensus-Verfahren diagnostiziert. Gleichermaßen wurde mit den Kontroll-CTs von Tag 1 und 3 sowie ggf. vor Entlassung verfahren. Anschließend wurden, zur Vergleichbarkeit der Daten, den Patienten mit cerebraler IPC bzw. ASSEinnahme jeweils ca. doppelt so viele Patienten ohne IPC / ASS gegenübergestellt. Patienten mit einer cerebralen IPC wiesen in dieser retrospektiven Studie eine signifikant niedrigere Nachblutungsrate auf, und bereits vorhandene Kontusionsblutungen zeigten seltener eine Größenprogredienz als bei jenen Patienten, die ohne eine solche Vorerkrankung das SHT erlitten (univariat $p<0.0001$ und multivariat $p=0.0007$ ). Hierbei wurden in der multivariaten Analyse Patientenalter, -geschlecht, Alkoholkonsum, ASS-Medikation und IPC berücksichtigt. Diese Ergebnisse sind vereinbar mit den initialen in-vivo-Ergebnissen im Tierversuch von Perez-Pinzon, welcher nach IPC geringere Volumina der erzeugten Kontusionsblutungen bei Ratten nachweisen konnte (Perez-Pinzon et al. 1999).

Obwohl die genauen Mechanismen der IPC noch nicht vollständig geklärt sind, aber valide Hinweise auf einen relevanten Einfluss dieses Faktors beim ischämischen Schlaganfall vorliegen (Masada et al. 2002; Moncayo et al. 2000; Weih et al. 1999), legen die limitierten Daten dieser Studie, zusammen mit den Ergebnissen im Tierversuch, nahe, dass eine IPC einen gewissen Schutz auch vor einem folgenden SHT darstellt (Perez-Pinzon et al. 1999). Während, ähnlich wie beim Schlaganfall, bei einem SHT ein plötzlicher Gewebeschaden unter anderem durch Minderperfusion im betroffenen Gebiet entsteht, kann eine IPC, die keine oder nur minimale Gewebeschäden hinterlässt, dazu beitragen, dass das Gehirn endogene Mechanismen ausbildet, die es vor einem folgenden SHT-induzierten neuronalen Schaden schützt. Die Hypothese einer perikontusionellen Sauerstoffminderversorgung wurde bereits in mehreren Studien untersucht. Hier führten beispielsweise in einer PET-Studie ein reduzierter Blutfluss sowie auch ein reduzierter Metabolismus in direkter Umgebung der Kontusionen nicht nur im akuten, sondern auch im subakuten Stadium zu chronischen Nervenzellschädigungen (Kawai et al. 2008). Ähnlich dem Modell der ischämischen Penumbra beim ischämischen Hirninfarkt weisen perikontusionelle Areale mit vermindertem Blutfluss 
auf ein Risiko für sekundäre Komplikationen beim SHT hin (Furuya et al. 2003; Orakcioglu et al. 2008; Schellinger et al. 2003; von Oettingen et al. 2002).

Bezüglich des zu untersuchenden, potentiell neuroprotektiven Faktors „ASSEinnahme" konnte in der univariaten Analyse ebenfalls ein signifikanter Unterschied in der Anzahl der Nachblutungen zugunsten der ASS Einnehmenden, welche eine geringere Nachblutungsrate aufwiesen, festgestellt werden $(p=0.0206)$. Multivariat unter Berücksichtigung der IPC - war dieses Ergebnis nicht aufrechtzuerhalten, da das Signifikanzniveau nicht erreicht werden konnte $(p=0.3780)$. Da die ASS Einnahme durch seine etablierte Medikation nach einer erfolgten TIA (Qureshi et al. 2009) stark mit dem Vorhandensein einer IPC korreliert, ist dieser Parameter nicht getrennt untersuchbar und in unserem Fall dementsprechend als nicht sicher neuroprotektiv zu werten. Dennoch existieren in der Literatur Hinweise auf eine verbesserte Hypoxietoleranz durch einen Wirkmechanismus der Acetylsalicylsäure. Hierbei wird zwischen der klassischen, ischämischen Episode und der milden, sogenannten chemischen Hypoxie unterschieden, welche beide zu einer verbesserten Hypoxietoleranz führen (Riepe et al. 1996b; Riepe et al. 1997b). Unabhängig von der Thrombozyten-aggregationshemmenden Wirkung verzögert eine Vorbehandlung mit ASS den Abfall des Energiemetabolismus und den ATPVerbrauch in Nervenzellen während einer ischämischen oder chemischen Hypoxie (Riepe et al. 1997a).

Bezüglich des GCS bei Aufnahme konnte beobachtet werden, dass, obwohl dieses tendenziell in der Gruppe der ischämisch Präkonditionierten und der ASS Einnehmenden um ca. einen Punkt höher ausfiel als in der Vergleichsgruppe, sich beide Gruppen bezüglich des GCS zum Entlassungszeitpunkt nicht signifikant unterschieden. Trotz verminderter Nachblutungsraten war das GCS nicht höher als in der Kontrollgruppe. Unter Berücksichtigung des Faktors, dass eine Patientenentlassung jedoch nach variierenden Liegezeiten erst nach Erreichen eines klinisch stabilen Zustandes erfolgt, wurde zusätzlich das GCS nach 3 Tagen korreliert. Hier waren ebenfalls keine signifikanten Unterschiede in beiden Patientengruppen erkennbar. Demnach korreliert eine Nachblutung also nicht zwingend mit einem schlechteren Patientenoutcome. Es konnte jedoch auch in dieser Studie die Tendenz erkannt werden, dass ein initial niedriges GCS öfter mit einer klinischen Verschlechterung (Ducrocq et al. 2006) und einer gering höheren 
Nachblutungsrate einhergeht. Es stellt sich außerdem die Frage, warum jüngere Patienten gemäß dieser Daten häufiger Nachblutungen erleiden, wobei ein möglicher Lösungsansatz hier eine vermehrte Prävalenz der IPC im höheren Lebensalter wäre. Zudem könnte ein Unterschied im Schweregrad der erlittenen Unfälle und ihrer zu Grunde liegenden Mechanismen einen Einfluss auf die Nachblutungsrate haben. Die hiesigen Daten zeigen zumindest ein signifikant niedrigeres Durchschnittsalter der Patienten nach PKW- und Motorradunfall im Vergleich zu Stürzen aus dem Stand, Synkopen und Treppenstürzen, welche ein Maximum zwischen der 6. und 7 . Lebensdekade aufweisen.

Die Ergebnisse dieser Studie zeigen weiterhin, dass Nachblutungen auch nach initial unauffälligem $\mathrm{CCT}$ in relevanten Prozentsätzen vorkommen, hierbei jedoch neben den bereits bekannten Entscheidungskriterien für ein follow-up-cCT wie neurologische Verschlechterung oder ansteigender Hirndruck (Brown et al. 2007; Oertel et al. 2002; Rieger et al. 2002) weitere, noch unbekannte Faktoren protektiv oder als Risikofaktor eine Rolle spielen. Da Nachblutungen anhand der limitierten Daten dieser Studie auch ohne signifikante, klinisch neurologische Veränderung auftreten können, unterstützt dieses Ergebniss wiederum die These einer perifokalen Minderdurchblutung, welche den präexistenten Funktionsverlust des Gewebes in der traumatischen Penumbra erklären könnte.

Die Theorie, dass das weibliche Geschlecht sich neuroprotektiv auswirken könnte, konnte in dieser Untersuchung nicht bestätigt werden, da die Nachblutungsrate bei beiden Geschlechtern ungefähr gleich war ( $33 \%$ w vs. $37 \% \mathrm{~m})$. Allerdings erlitten in diesem Zeitraum ca. doppelt so viele Männer wie Frauen ein SHT.

In dieser retrospektiven Fallkontrollstudie sind fehlende Informationen durch unvollständige Dokumentationen in den Patientenakten nicht auszuschließen. So wäre es möglich, dass etwa relevante Vorerkrankungen nicht dokumentiert wurden bzw. vom Patienten nicht wahrgenommen wurden. Dies gilt bezüglich der IPC eher für einzelne Patienten der nicht präkonditionierten Gruppe, somit sollte durch die größere Fallzahl keine statistisch relevante Beeinflussung verursacht werden. Da das Universitätsklinikum Göttingen allerdings über die einzige neurologische Notaufnahme und Stroke-Unit im größeren Einzugsgebiet verfügt, ist davon auszugehen, dass die Mehrheit der TIA-Patienten hier eingeliefert werden und somit wahrgenommene, klinisch relevante TIAs bekannt sein dürften. Die Aufnahme in ein 
Universitätsklinikum stellt einen bekannten potentiell selektionsbedingten und verzerrenden Fehler dar und kann auch in dieser Studie nicht mit Sicherheit ausgeschlossen werden: Als Haus der Maximalversorgung ist es auf Grund von Patientenverlegungen möglich, dass die ausgewählten SHT-Patienten nicht repräsentativ für eine größere Population sind und schwere SHTs überproportional häufig vorkommen.

Bezüglich der erhobenen Daten der potentiell neuroprotektiven Faktoren müssen, neben der o. g. Korrelation der IPC mit der ASS Medikation, weitere Einschränkungen berücksichtigt werden: weder für ASS noch für die IPC kann hier ein einheitliches Schema festgelegt werden. Zu welchem Zeitpunkt vor dem Unfall und über welche Dauer ASS von den Patienten eingenommen wurde, ist nicht bekannt, ebenso wenig wie oft Patienten eine TIA erlitten bzw. wie schwer die Symptomatik einer solchen war. Bezüglich der Dosis standen die Patienten überwiegend unter Low-Dose-ASS-Therapie $(100 \mathrm{mg} / \mathrm{d})$. Allerdings konnte in bisherigen Studien keine Korrelation zwischen dem Schweregrad des Stimulus und erfolgter Neuroprotektion nachgewiesen werden, so dass bislang eher ein Ja/NeinPrinzip gilt und eine individualisierte Dosisanpassung empfohlen wird (Ansara et al. 2010). Mikroangiopathische Veränderungen des Gehirns im CCT wurden ebenso nur als vorhanden oder nicht vorhanden aufgenommen, der Schweregrad also nicht berücksichtigt. $\mathrm{Da}$ in dieser Studie jedoch lediglich Anhaltspunkte für einen möglichen neuroprotektiven Effekt der untersuchten Faktoren gesucht werden sollten, kann dieser Punkt vernachlässigt werden. Eine Quantifizierung der Ergebnisse war nicht das Ziel der Untersuchung.

Zusammenfassend, trotz der hier angeführten Unsicherheitsfaktoren, deuten die vorliegenden Daten darauf hin, dass eine cerebrale IPC einen neuroprotektiven Effekt auf das Hirngewebe hat, möglicherweise verursacht durch eine verbesserte endogene Hypoxietoleranz. Diese Theorie kann durch oben genannte Studie von Perez-Pinzon et al. (Perez-Pinzon et al. 1999) gestützt werden. Darüber hinaus kann durch die vorliegenden Daten die These unterstrichen werden, dass follow-up-CTs auch bei initial unauffälligem CT notwendig sind, da die prognostische Aussagekraft des initialen CTs bezüglich des klinischen Verlaufs eines SHT-Patienten fraglich ist. Nachblutungen kommen auch bei Patienten mit zunächst unauffälligem $\mathrm{CCT}$ und 
GCS mit einer Latenz von Stunden bis Tagen vor und entgehen somit dem initialen CT. 


\section{Zusammenfassung}

Der endogene adaptive Mechanismus der Hypoxietoleranzentwicklung, als ischemic tolerance bezeichnet, wurde sowohl für Angina-pectoris-Anfälle vor einem folgenden Myokardinfarkt als auch für eine TIA vor einem ischämischen Hirninfarkt beschrieben. Da es Anhalte dafür gibt, dass eine Minderperfusion des Hirngewebes ebenfalls beim SHT eine Rolle zu spielen scheint, sollte in dieser retrospektiven Studie untersucht werden, ob eine cerebrale IPC in Form einer TIA vor dem Trauma oder mikroangiopathische Veränderungen des Hirnparenchyms im CT oder die als neuroprotektiv beschriebene Acetylsalicylsäure eine positive Auswirkung auf die Nachblutungsrate bei SHT-Patienten haben.

Es zeigten sich signifikante Unterschiede der beiden Gruppen bezüglich der Nachblutungsrate bei den ischämisch präkonditionierten und auch bei den ASS einnehmenden Patienten (primärer Endpunkt): Die Nachblutungsrate betrug in der Gruppe der ischämisch präkonditionierten 10,4\% und in der Vergleichsgruppe 48,9 $\% \quad(p<0,001)$. In der Gruppe der ASS Einnehmenden zeigte sich eine Nachblutungsrate von 19,5\%, während die Kontrollgruppe in $42,4 \%$ eine Nachblutung aufwies. In der multivarianten Analyse korrelierte die ASS Einnahme jedoch stark mit der IPC, die Ergebnisse erreichten hier nicht mehr das Signifikanzniveau.

Der Glasgow Coma Scale als Maß für das neurologische Defizit bei Entlassung unterschied sich nicht signifikant (12,9 bei den ischämisch präkonditionierten und 12,7 in der Kontrollgruppe bzw. 12,9 in der ASS-Gruppe und 12,4 in der Vergleichsgruppe).

Insgesamt traten bei $35,7 \%$ der Patienten Nachblutungen auf, bei initial unauffälligem CT sogar in 54 \% (22/41) der Fälle.

Die Ergebnisse dieser Studie legen nahe, dass eine IPC vor einem SHT eine Rolle spielen könnte. Da jedoch die vorliegenden Daten zeigen, dass eine Nachblutung nicht unbedingt mit einem schlechteren neurologischen Outcome einhergeht, müssen noch andere Faktoren existieren, die den klinischen Verlauf eines SHTPatienten beeinflussen. Diese Studie belegt erneut die Notwendigkeit von Verlaufskontrollen - z.B. mittels CT - da auch bei initial unauffälligem CT-Befund intraparenchymale Blutungen auftreten. Die Tatsache, dass dies anscheinend ohne 
wesentliche klinische Veränderung stattfinden kann, stützt erneut die These der perifokalen Minderdurchblutung, da diese den Funktionsverlust erklären könnte. 


\section{Literaturverzeichnis}

Akdemir Ozisik P, Oruckaptan H, Ozdemir Geyik P, Misirlioglu M, Sargon MF, Kilinc K, Ozgen T (2007): Effect of erythropoietin on brain tissue after experimental head trauma in rats. Surg Neurol $\underline{68}, 547-55$; discussion 555

Andreotti F, Pasceri V, Hackett DR, Davies GJ, Haider AW, Maseri A (1996): Preinfarction angina as a predictor of more rapid coronary thrombolysis in patients with acute myocardial infarction. N Engl J Med $\underline{334}, 7-12$

Ansara AJ, Nisly SA, Arif SA, Koehler JM, Nordmeyer ST (2010): Aspirin dosing for the prevention and treatment of ischemic stroke: an indication-specific review of the literature. Ann Pharmacother 444, 851-62

Baumgartner U, Baier PK, Schmitz DT, Farthmann EH (2002): [Brain concussion: risk factors]. Swiss Surg $\underline{8}, 259-65$

Berry C, Ley EJ, Tillou A, Cryer G, Margulies DR, Salim A (2009): The effect of gender on patients with moderate to severe head injuries. J Trauma $\underline{67}$, 950-3

Biberthaler P, Mussack T, Wiedemann E, Kanz KG, Koelsch M, Gippner-Steppert C, Jochum M (2001): Evaluation of $S-100 b$ as a specific marker for neuronal damage due to minor head trauma. World J Surg $\underline{25}$, 93-7

Bramlett HM, Dietrich WD (2001): Neuropathological protection after traumatic brain injury in intact female rats versus males or ovariectomized females. J Neurotrauma 18, 891-900

Brown CV, Zada G, Salim A, Inaba K, Kasotakis G, Hadjizacharia P, Demetriades D, Rhee $P$ (2007): Indications for routine repeat head computed tomography (CT) stratified by severity of traumatic brain injury. J Trauma 62, 1339-44; discussion 1344-5

Cortbus F, Steudel WI (1999): Internationale und nationale Daten zur Epidemiologie des Schädelhirntraumas. Klinik \& Forschung $\underline{\text { S1, 4-10 }}$ 
Cutler SM, Cekic M, Miller DM, Wali B, VanLandingham JW, Stein DG (2007): Progesterone improves acute recovery after traumatic brain injury in the aged rat. J Neurotrauma $\underline{24}, 1475-86$

Dave KR, Saul I, Busto R, Ginsberg MD, Sick TJ, Perez-Pinzon MA (2001): Ischemic preconditioning preserves mitochondrial function after global cerebral ischemia in rat hippocampus. $\mathrm{J}$ Cereb Blood Flow Metab 21, 1401-10

Dihanich M, Kaser M, Reinhard E, Cunningham D, Monard D (1991): Prothrombin mRNA is expressed by cells of the nervous system. Neuron $\underline{6}, 575-81$

Ducrocq SC, Meyer PG, Orliaguet GA, Blanot S, Laurent-Vannier A, Renier D, Carli PA (2006): Epidemiology and early predictive factors of mortality and outcome in children with traumatic severe brain injury: experience of a French pediatric trauma center. Pediatr Crit Care Med $\underline{7}$, 461-7

Durukan A, Tatlisumak T (2010): Preconditioning-induced ischemic tolerance: a window into endogenous gearing for cerebroprotection. Exp Transl Stroke Med $\underline{2}, 2$

Ehrenreich H, Hasselblatt M, Dembowski C, Cepek L, Lewczuk P, Stiefel M, Rustenbeck HH, Breiter N, Jacob S, Knerlich Fet al. (2002): Erythropoietin therapy for acute stroke is both safe and beneficial. Mol Med $\underline{8}, 495-505$

Ehrenreich H, Weissenborn K, Prange H, Schneider D, Weimar C, Wartenberg K, Schellinger PD, Bohn M, Becker H, Wegrzyn Met al. (2009): Recombinant human erythropoietin in the treatment of acute ischemic stroke. Stroke $\underline{40}$, e647-56

Eriskat J, Stoffel M, Plesnila N, Rinecker M, Baethmann A (2000): Interstitial lactate in the peritraumatic penumbra of rat brain. Acta Neurochir Suppl $\underline{76}, 353-5$

Evrengul H, Kayikcioglu M, Can L, Payzin S, Kultursay H (2003): Effect of preinfarction angina on ventricular late potentials in patients with acute myocardial infarction and successful thrombolysis. Acta Cardiol $\underline{58}, 295-301$ 
Friedrich DH, Plesnila N, Eriskat J, Stoffel M, Baethmann A (2000): Cerebral blood flow and the secondary growth of brain tissue necrosis after trauma. Acta Neurochir Suppl $\underline{76}, 257-9$

Furuya Y, Hlatky R, Valadka AB, Diaz P, Robertson CS (2003): Comparison of cerebral blood flow in computed tomographic hypodense areas of the brain in head-injured patients. Neurosurgery $\underline{52}, 340-5$; discussion 345-6

Grilli M, Pizzi M, Memo M, Spano P (1996): Neuroprotection by aspirin and sodium salicylate through blockade of NF-kappaB activation. Science $\underline{274}, 1383-5$

Haacke EM, Xu Y, Cheng YC, Reichenbach JR (2004): Susceptibility weighted imaging (SWI). Magn Reson Med 르, 612-8

Haacke EM, Mittal S, Wu Z, Neelavalli J, Cheng YC (2009): Susceptibility-weighted imaging: technical aspects and clinical applications, part 1. AJNR Am J Neuroradiol $\underline{30}, 19-30$

Hamada R, Matsuoka H (2000): Antithrombin therapy for intracerebral hemorrhage. Stroke $\underline{31}, 794-5$

Hamilton M, Mrazik M, Johnson DW (2010): Incidence of delayed intracranial hemorrhage in children after uncomplicated minor head injuries. Pediatrics $\underline{126}, \mathrm{e} 33-9$

Herweh C, Juttler E, Schellinger PD, Klotz E, Jenetzky E, Orakcioglu B, Sartor K, Schramm $P$ (2007): Evidence against a perihemorrhagic penumbra provided by perfusion computed tomography. Stroke $\underline{38}, 2941-7$

Heusch G (2001): Nitroglycerin and delayed preconditioning in humans: yet another new mechanism for an old drug? Circulation $\underline{103}, 2876-8$

Heusch G, Schulz R (1997): Endogenous protective mechanisms in myocardial ischemia: hibernation and ischemic preconditioning. Am J Cardiol 80, 26A-33A 
Hurn PD, Littleton-Kearney MT, Kirsch JR, Dharmarajan AM, Traystman RJ (1995): Postischemic cerebral blood flow recovery in the female: effect of 17 betaestradiol. J Cereb Blood Flow Metab 15, 666-72

Jiang Y, Wu J, Hua Y, Keep RF, Xiang J, Hoff JT, Xi G (2002): Thrombin-receptor activation and thrombin-induced brain tolerance. J Cereb Blood Flow Metab $\underline{22}, 404-10$

Kawai N, Nakamura T, Tamiya T, Nagao S (2008): Metabolic disturbance without brain ischemia in traumatic brain injury: a positron emission tomography study. Acta Neurochir Suppl 102, 241-5

Kennedy DM, Cipolle MD, Pasquale MD, Wasser T (2000): Impact of preinjury warfarin use in elderly trauma patients. J Trauma $\underline{48}, 451-3$

Koch G (1996): Unfallursachen beim Schädel-Hirn-Trauma. Oberhessische Presse 28.03.1996, $12-13$

Lee KR, Betz AL, Kim S, Keep RF, Hoff JT (1996a): The role of the coagulation cascade in brain edema formation after intracerebral hemorrhage. Acta Neurochir (Wien) 138, 396-400; discussion 400-1

Lee KR, Colon GP, Betz AL, Keep RF, Kim S, Hoff JT (1996b): Edema from intracerebral hemorrhage: the role of thrombin. J Neurosurg $\underline{84}, 91-6$

Liu AY, Maldjian JA, Bagley LJ, Sinson GP, Grossman RI (1999): Traumatic brain injury: diffusion-weighted MR imaging findings. AJNR Am J Neuroradiol $\underline{20}$, 1636-41

Lobato RD, Rivas JJ, Gomez PA, Castaneda M, Canizal JM, Sarabia R, Cabrera A, Munoz MJ (1991): Head-injured patients who talk and deteriorate into coma. Analysis of 211 cases studied with computerized tomography. J Neurosurg $\underline{75}$, 256-61

Lu D, Mahmood A, Qu C, Goussev A, Schallert T, Chopp M (2005): Erythropoietin 
enhances neurogenesis and restores spatial memory in rats after traumatic brain injury. J Neurotrauma $\underline{22}, 1011-7$

Masada T, Xi G, Hua Y, Keep RF (2000): The effects of thrombin preconditioning on focal cerebral ischemia in rats. Brain Res $\underline{867}, 173-9$

Masada T, Hua Y, Xi G, Ennis SR, Keep RF (2002): Effect of ischemic preconditioning on edema formation and cerebrovascular injury following focal cerebral ischemia. Acta Neurochir Suppl $\underline{81}$, 265-8

Mattioli C, Beretta L, Gerevini S, Veglia F, Citerio G, Cormio M, Stocchetti N (2003): Traumatic subarachnoid hemorrhage on the computerized tomography scan obtained at admission: a multicenter assessment of the accuracy of diagnosis and the potential impact on patient outcome. J Neurosurg $\underline{98}, 37-42$

Mayer SA, Coplin WM, Raps EC (1999): Cerebral edema, intracranial pressure, and herniation syndromes. J Stroke Cerebrovasc Dis $\underline{8}, 183-191$

McBride DQ, Patel AB, Caron M (1993): Early repeat CT scan: importance in detecting surgical lesions after closed head injury. J Neurotrauma 10 (Suppl 1), 227

Meldrum B (1990): Protection against ischaemic neuronal damage by drugs acting on excitatory neurotransmission. Cerebrovasc Brain Metab Rev 2 2, 27-57

Menon DK (2003): Procrustes, the traumatic penumbra, and perfusion pressure targets in closed head injury. Anesthesiology $\underline{98}, 805-7$

Mina AA, Knipfer JF, Park DY, Bair HA, Howells GA, Bendick PJ (2002): Intracranial complications of preinjury anticoagulation in trauma patients with head injury. J Trauma $\underline{53}$, 668-72

Mina AA, Bair HA, Howells GA, Bendick PJ (2003): Complications of preinjury warfarin use in the trauma patient. J Trauma $\underline{54}, 842-7$ 
Moncayo J, de Freitas GR, Bogousslavsky J, Altieri M, van Melle G (2000): Do transient ischemic attacks have a neuroprotective effect? Neurology $\underline{54}, 2089-$ 94

Murry CE, Jennings RB, Reimer KA (1986): Preconditioning with ischemia: a delay of lethal cell injury in ischemic myocardium. Circulation $\underline{74}, 1124-36$

Mushkudiani NA, Engel DC, Steyerberg EW, Butcher I, Lu J, Marmarou A, Slieker F, McHugh GS, Murray GD, Maas Al (2007): Prognostic value of demographic characteristics in traumatic brain injury: results from the IMPACT study. J Neurotrauma 24, 259-69

Nellgard B, Wieloch T (1992): Postischemic blockade of AMPA but not NMDA receptors mitigates neuronal damage in the rat brain following transient severe cerebral ischemia. J Cereb Blood Flow Metab 12, 2-11

Nirula R, Diaz-Arrastia R, Brasel K, Weigelt JA, Waxman K (2010): Safety and efficacy of erythropoietin in traumatic brain injury patients: a pilot randomized trial. Crit Care Res Pract $\underline{2010}$

Nordstrom CH, Reinstrup P, Xu W, Gardenfors A, Ungerstedt U (2003): Assessment of the lower limit for cerebral perfusion pressure in severe head injuries by bedside monitoring of regional energy metabolism. Anesthesiology $\underline{98}, 809-14$

Oertel M, Kelly DF, McArthur D, Boscardin WJ, Glenn TC, Lee JH, Gravori T, Obukhov D, McBride DQ, Martin NA (2002): Progressive hemorrhage after head trauma: predictors and consequences of the evolving injury. J Neurosurg 96, $109-16$

Orakcioglu B, Becker K, Sakowitz OW, Unterberg A, Schellinger PD (2008): Serial diffusion and perfusion MRI analysis of the perihemorrhagic zone in a rat $\mathrm{ICH}$ model. Acta Neurochir Suppl 103, 15-8

Ottani F, Galvani M, Ferrini D, Sorbello F, Limonetti P, Pantoli D, Rusticali F (1995): Prodromal angina limits infarct size. A role for ischemic preconditioning. 
Circulation $\underline{91}, 291-7$

Perez-Pinzon MA, Alonso O, Kraydieh S, Dietrich WD (1999): Induction of tolerance against traumatic brain injury by ischemic preconditioning. Neuroreport $\underline{10}$, 2951-4

Pettus EH, Wright DW, Stein DG, Hoffman SW (2005): Progesterone treatment inhibits the inflammatory agents that accompany traumatic brain injury. Brain Res $\underline{1049}, 112-9$

Phelan HA, Shafi S, Parks J, Maxson RT, Ahmad N, Murphy JT, Minei JP (2007): Use of a pediatric cohort to examine gender and sex hormone influences on outcome after trauma. J Trauma $\underline{63}, 1127-31$

Pieracci FM, Eachempati SR, Shou J, Hydo LJ, Barie PS (2007): Degree of anticoagulation, but not warfarin use itself, predicts adverse outcomes after traumatic brain injury in elderly trauma patients. J Trauma $\underline{63}, 525-30$

Qureshi Al, Feldmann E, Gomez CR, Johnston SC, Kasner SE, Quick DC, Rasmussen PA, Suri MF, Taylor RA, Zaidat OO (2009): Consensus conference on intracranial atherosclerotic disease: rationale, methodology, and results. J Neuroimaging 19 Suppl 1 , 1S-10S

Racay P, Tatarkova Z, Drgova A, Kaplan P, Dobrota D (2007): Effect of ischemic preconditioning on mitochondrial dysfunction and mitochondrial p53 translocation after transient global cerebral ischemia in rats. Neurochem Res $\underline{32}, 1823-32$

Rieger J, Linsenmaier U, Pfeifer KJ, Reiser M (2002): [Radiological diagnosis in acute craniocerebral trauma]. Radiologe $\underline{42}, 547-55$

Riepe MW, Niemi WN, Megow D, Ludolph AC, Carpenter DO (1996a): Mitochondrial oxidation in rat hippocampus can be preconditioned by selective chemical inhibition of succinic dehydrogenase. Exp Neurol 138, 15-21 
Riepe MW, Kasischke K, Gericke CA, Lowe A, Hellweg R (1996b): Increase of hypoxic tolerance in rat hippocampal slices following 3-nitropropionic acid is not mediated by endogenous nerve growth factor. Neurosci Lett 211, 9-12

Riepe MW, Kasischke K, Raupach A (1997a): Acetylsalicylic acid increases tolerance against hypoxic and chemical hypoxia. Stroke $\underline{28}, 2006-11$

Riepe MW, Esclaire F, Kasischke K, Schreiber S, Nakase H, Kempski O, Ludolph AC, Dirnagl U, Hugon J (1997b): Increased hypoxic tolerance by chemical inhibition of oxidative phosphorylation: "chemical preconditioning". J Cereb Blood Flow Metab 17, 257-64

Robertson CL, Puskar A, Hoffman GE, Murphy AZ, Saraswati M, Fiskum G (2006): Physiologic progesterone reduces mitochondrial dysfunction and hippocampal cell loss after traumatic brain injury in female rats. Exp Neurol 197, 235-43

Roof RL, Hall ED (2000): Gender differences in acute CNS trauma and stroke: neuroprotective effects of estrogen and progesterone. J Neurotrauma 17, 36788

Roof RL, Duvdevani R, Heyburn JW, Stein DG (1996): Progesterone rapidly decreases brain edema: treatment delayed up to 24 hours is still effective. Exp Neurol $\underline{138}, 246-51$

Saboori M, Ahmadi J, Farajzadegan Z (2007): Indications for brain CT scan in patients with minor head injury. Clin Neurol Neurosurg $\underline{109}$, 399-405

Schellinger PD, Fiebach JB, Hoffmann K, Becker K, Orakcioglu B, Kollmar R, Juttler E, Schramm P, Schwab S, Sartor Ket al. (2003): Stroke MRI in intracerebral hemorrhage: is there a perihemorrhagic penumbra? Stroke $\underline{34}, 1674-9$

Schnellinger MG, Reid S, Louie J (2010): Are serial brain imaging scans required for children who have suffered acute intracranial injury secondary to blunt head trauma? Clin Pediatr (Phila) $\underline{49}, 569-73$ 
Schouten JW (2007): Neuroprotection in traumatic brain injury: a complex struggle against the biology of nature. Curr Opin Crit Care $\underline{13}, 134-42$

Schutzman SA, Barnes PD, Mantello M, Scott RM (1993): Epidural hematomas in children. Ann Emerg Med 22, 535-41

Servadei F, Nasi MT, Giuliani G, Cremonini AM, Cenni P, Zappi D, Taylor GS (2000): CT prognostic factors in acute subdural haematomas: the value of the 'worst' CT scan. Br J Neurosurg 14, 110-6

Shikamoto $Y$, Morita $T$ (1999): Expression of factor $X$ in both the rat brain and cells of the central nervous system. FEBS Lett $\underline{463}, 387-9$

Simon RP, Swan JH, Griffiths T, Meldrum BS (1984): Blockade of N-methyl-Daspartate receptors may protect against ischemic damage in the brain. Science $\underline{226}, 850-2$

Slewa-Younan S, Baguley IJ, Heriseanu R, Cameron ID, Pitsiavas V, Mudaliar Y, Nayyar V (2008): Do men and women differ in their course following traumatic brain injury? A preliminary prospective investigation of early outcome. Brain Inj 22, $183-91$

Spektor S, Agus S, Merkin V, Constantini S (2003): Low-dose aspirin prophylaxis and risk of intracranial hemorrhage in patients older than 60 years of age with mild or moderate head injury: a prospective study. J Neurosurg $\underline{99}, 661-5$

Stein DG, Hoffman SW (2003): Estrogen and progesterone as neuroprotective agents in the treatment of acute brain injuries. Pediatr Rehabil $\underline{6}, 13-22$

Stein SC, Young GS, Talucci RC, Greenbaum BH, Ross SE (1992): Delayed brain injury after head trauma: significance of coagulopathy. Neurosurgery $\underline{30}, 160-$ 5

Stein SC, Spettell C, Young G, Ross SE (1993): Delayed and progressive brain injury in closed-head trauma: radiological demonstration. Neurosurgery $\underline{32}, 25-30$; 
discussion 30-1

Steudel WI, Cortbus F, Schwerdtfeger K (2005): Epidemiology and prevention of fatal head injuries in Germany--trends and the impact of the reunification. Acta Neurochir (Wien) 147, 231-42; discussion 242

Stiell IG, Lesiuk H, Wells GA, McKnight RD, Brison R, Clement C, Eisenhauer MA, Greenberg GH, MacPhail I, Reardon Met al. (2001): The Canadian CT Head Rule Study for patients with minor head injury: rationale, objectives, and methodology for phase I (derivation). Ann Emerg Med $\underline{38}, 160-9$

Theysohn JM, Kraff O, Maderwald S, Barth M, Ladd SC, Forsting M, Ladd ME, Gizewski ER (2011): 7 tesla MRI of microbleeds and white matter lesions as seen in vascular dementia. J Magn Reson Imaging 33, 782-91

Topal NB, Hakyemez B, Erdogan C, Bulut M, Koksal O, Akkose S, Dogan S, Parlak $M$, Ozguc H, Korfali E (2008): MR imaging in the detection of diffuse axonal injury with mild traumatic brain injury. Neurol Res $\underline{30}, 974-8$

von Oettingen G, Bergholt B, Gyldensted C, Astrup J (2002): Blood flow and ischemia within traumatic cerebral contusions. Neurosurgery $\underline{50}, 781-8$; discussion 788-90

Wagner AK, Willard LA, Kline AE, Wenger MK, Bolinger BD, Ren D, Zafonte RD, Dixon CE (2004): Evaluation of estrous cycle stage and gender on behavioral outcome after experimental traumatic brain injury. Brain Res $\underline{998}, 113-21$

Weih M, Kallenberg K, Bergk A, Dirnagl U, Harms L, Wernecke KD, Einhaupl KM (1999): Attenuated stroke severity after prodromal TIA: a role for ischemic tolerance in the brain? Stroke $\underline{30}, 1851-4$

Wiesmann M, Bruckmann H (1998): [Diagnostic imaging of acute head and brain injuries]. Radiologe $\underline{38}, 645-58$

Wilterdink JL, Furie KL, Easton JD (1998): Cardiac evaluation of stroke patients. 
Neurology $\underline{51}$, S23-6

Wojcik R, Cipolle MD, Seislove E, Wasser TE, Pasquale MD (2001): Preinjury

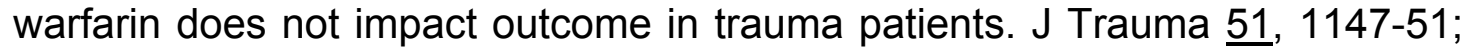
discussion 1151-2

Wu X, Hu J, Zhuo L, Fu C, Hui G, Wang Y, Yang W, Teng L, Lu S, Xu G (2008): Epidemiology of traumatic brain injury in eastern China, 2004: a prospective large case study. J Trauma $\underline{64}, 1313-9$

Xi G, Keep RF, Hua Y, Xiang J, Hoff JT (1999): Attenuation of thrombin-induced brain edema by cerebral thrombin preconditioning. Stroke $\underline{30}, 1247-55$

Xi G, Keep RF, Hua Y, Hoff JT (2000): Thrombin preconditioning, heat shock proteins and thrombin-induced brain edema. Acta Neurochir Suppl $\underline{76}, 511-5$

Xi G, Hua Y, Wu J, Keep RF (2002): Increase of brain thrombin concentration in cerebral ischemia. Stroke $\underline{33}, 399$

Xi G, Reiser G, Keep RF (2003): The role of thrombin and thrombin receptors in ischemic, hemorrhagic and traumatic brain injury: deleterious or protective? $\mathrm{J}$ Neurochem $\underline{84}, 3-9$

Yellon DM, Baxter GF, Garcia-Dorado D, Heusch G, Sumeray MS (1998): Ischaemic preconditioning: present position and future directions. Cardiovasc Res $\underline{37}, 21-$ 33

Zazulia AR, Diringer MN, Videen TO, Adams RE, Yundt K, Aiyagari V, Grubb RL, Jr., Powers WJ (2001): Hypoperfusion without ischemia surrounding acute intracerebral hemorrhage. J Cereb Blood Flow Metab 21, 804-10

Zhan RZ, Fujihara H, Baba H, Yamakura T, Shimoji K (2002): Ischemic preconditioning is capable of inducing mitochondrial tolerance in the rat brain. Anesthesiology $\underline{97}$, 896-901 
Zhan X, Ander BP, Liao IH, Hansen JE, Kim C, Clements D, Weisbart RH, Nishimura RN, Sharp FR (2010): Recombinant Fv-Hsp70 protein mediates neuroprotection after focal cerebral ischemia in rats. Stroke $\underline{41}, 538-43$

Zimmer A, Reith W (2008): [Diagnostic imaging of traumatic brain injury.]. Radiologe $\underline{48}, 503-18$ 


\section{Abkürzungen}

A. Arteria (lat., Arterie)

Aa. Arteriae (lat., Arterien)

AP Angina pectoris

ASS Acetylsalicylsäure

ATP Adenosintriphosphat

cCT kraniale Computertomographie

CT Computertomographie

DAI diffuse axonal injury

DWI diffusion weighted imaging

EDH Epiduralhämatom

GCS Glasgow Coma Scale

HSP Hitzeschockprotein

HOPS Hirnorganisches Psychosyndrom

ICB intracerebrale Blutung

INR international normalized ratio

IPC ischemic preconditioning

mRNA messenger ribonucleic acid

MRS modified ranking scale

MRT Magnetresonanztomographie

$\mathrm{O}_{2} \quad$ Sauerstoff

PAI Plasminogen-Aktivator-Inhibitor

PET Positronenemissionstomographie

pTT partielle Thromboplastinzeit

$\mathrm{PWI} \quad$ perfusion weighted imaging

SD standard deviation

SDH Subduralhämatom

SHT Schädelhirntrauma

TIA transitorische ischämische Attacke

tSAB traumatische Subarachnoidalblutung

T/N Tag-/Nachtaufnahme

UCLA University of California, Los Angeles 
10. Anhang

Anlage 1:

SHT-Fragebogen

Fragebogen:

ID:

Geburtsjahr:

Traumahergang:

Sturz:

MVA:

Radunfall:

Sonstiges:

Unfallsdatum / Uhrzeit:

Datum / Uhrzeit der Notambulanzankunft:

CT Notambulanz:

Aufnahme: nein ja

Normal-Station

Intensivstation

Aufnahmeparameter:

RR:

Krea: 
Comascale:

$\begin{array}{lll}\text { Übelkeit: } & \text { nein } & \text { ja } \\ \text { Erbrechen: nein } & \text { ja } \\ \text { Visuelle Symptome: } & \text { nein ja }\end{array}$

CT: $\quad$ 1. initiale CT (Dat. / Uhrzeit / Befund)

2. follow - up CT (s.o.)

nach 1d:

nach $3 \mathrm{~d}$ u./o. E:
GCS:
1. Aufnahme:
MRS (min. 12 Mon. Bis Ende offen):
2. nach $1 \mathrm{~d}$ :
3. nach 3d u. / O. E:

Komplikationen:

$\begin{array}{ll}\text { Infektionen nein } & \text { ja } \\ \text { Nachblutung nein } & \text { ja } \\ \text { Sonstiges nein } & \text { ja }\end{array}$

Tod 
Vorerkrankungen:

1. Herz / Kreislauf: HRST KHK PM/Klappe HTN

AP $\quad$ MI

2. Sonstige: DM Niere Nikotin

Stroke TIA Stoffwechselerkrankungen

Alkohol

Vormedikation:

$\beta$ - Blocker $\quad$ EPO

ASS / Plavix Östrogen/Progesteron

Marcumar Glibenclamide

Immunsuppressiva sonstige 
Anlage 2:

\section{Glasgow Coma Scale}

Glasgow Coma Scale

\begin{tabular}{ccll}
\hline Punkte & Augen öffnen & \multicolumn{1}{c}{ Verbale Kommunikation } & \multicolumn{1}{c}{ Motorische Reaktion } \\
\hline 6 & - & - & befolgt Aufforderungen \\
5 & - & konversationsfähig, orientiert & gezielte Schmerzabwehr \\
4 & spontan & konversationsfähig, desorientiert & ungezielte Schmerzabwehr \\
3 & auf Aufforderung & unzusammenhängende Worte & auf Schmerzreiz Beugesynergismen \\
2 & auf Schmerzreiz & unverständliche Laute & auf Schmerzreiz Strecksynergismen \\
1 & keine Reaktion & keine verbale Reaktion & keine Reaktion auf Schmerzreiz \\
\hline
\end{tabular}

Schweregrad: Leicht: 14-15 Punkte ; Mittel: 9-13 Punkte ; Schwer: 3-8 Punkte 